\begin{tabular}{|c|c|c|c|}
\hline $\begin{array}{c}\text { Travaux du Muséum National d'Histoire Naturelle } \\
\text { «Grigore Antipa» }\end{array}$ & Vol. LIV (1) & pp. 243-262 & $\begin{array}{c}\text { (C) 30 Juin } \\
2011\end{array}$ \\
\hline
\end{tabular}

DOI: 10.2478/v10191-011-0015-1

\title{
MICROEVOLUTION OF BECHSTEIN'S BAT MYOTIS BECHSTEINII (KUHL, 1817) (MAMMALIA: CHIROPTERA) IN THE HOLOCENE OF SOUTHERN POLAND
}

\author{
KATARZYNA STANIK, BRONISŁAW W. WOŁOSZYN
}

\begin{abstract}
Bechstein's bat was one of the most common species in the Middle and Late Holocene of Southern Poland. It dominated in Kraków - Czestochowa Upland, but was also frequent in Tatra Mountains. Bone material from thanatocoenoses, dated by ${ }^{14} \mathrm{C}$ AMS method, was analysed. The aim of studies was to investigate the direction of changes in skull and mandible morphology of Myotis bechsteinii during the period from $5710 \mathrm{BC}$ till present day and to answer the question which environmental parameters significantly correlates with these microevolutionary processes and frequency of species in different thanatocoenoses. Mandibles and skulls were analysed separately. Morphology was correlated with climate characteristics, temperatures, and percentage pollen of such species of trees as hornbeam (Carpinus betulus), beech (Fagus sylvatica), oak (Quercus) and elm (Ulmus) in investigated time limits.

Résumé. On a étudié les tendances microévolutives du crâne de la chauve-souris de Bechstein. Malgré le fait que les différences entre les groupes ne sont pas remarquables du point de vue statistique, les tendances microévolutives morphologiques sont visibles. Les changements de chaque paramètre ont un cours individuel, mais les tendances sont à peu près similaires. L'analyse statistique a rendue possible la réponse à la question quels facteurs de niche ont une influence importante sur la morphologie du crâne et quelle est la direction de ces influences. La température moyenne du mois de janvier, le pourcentage de Fraxinus excelsior, la fréquence de la chauve-souris de Bechstein et la fréquence des inondations déterminent la majeure partie des changements morphologiques.
\end{abstract}

Key words: Holocene, Bechstein's bat, microevolution, Southern Poland.

\section{INTRODUCTION}

Holocene is the last warm period of Quaternary, with the beginning dated to $10200{ }^{14} \mathrm{C}$ BP (Starkel, 1999). In this relatively short period, many fluctuations in climate and changes in vegetation (Cheddadi \& Bar-Hen, 2009; Raible et al., 2006; Ralska-Jasiewiczowa et al., 2004) occured. Holocene is the best known geological period, in which present flora and fauna was moulded on the North of the Carpathian Mountains (Pawłowski, 1999).

Bechstein's bat is actually restricted to southern and central Poland and considered rare in the country. While during the Atlantic and Subboreal period its frequency in thanatocoenoses varied from $2-65 \%$, it have been almost completely eliminated from recent fauna of Polish Tatras (Postawa, 2004; Piksa \& Wołoszyn, 2001). Forest complexes dominated by old hollowed decidous trees are typical natural biotops of this species. In the diet of Myotis bechsteinii (Kuhl, 1817) moths, beetles, spiders etc. dominate, catched mostly low between dense thickets (Beck, 1995). It inhabitates similar ecologycal niche as Myotis nattereri (Fenton \& Bogdanowicz, 2002). If the competition for the best habitat is significant between these two species, it can have influence on their microevolution.

Morphology of population can be modified by age and sex structure (Krebs, 2001), despite the fact that investigated subfossil remains are from winter 
hibernacula (Postawa, 2004; Piksa \& Wołoszyn, 2001), and sexual dimorphism of small bats is not evident (Bogdanowicz, 1992). Analysis of Bechstein's bat recent population makes possible the investigation of these problems.

The aim of this study is to investigate microevolutionary trends in cranial skeleton morphology of Bechstein's bat and to answer to the question which of several factors (such as temperature, hight above sea level, habitat structure) have significant influence on the morphology and which is the direction of this influence (positive or negative correlation).

\section{MATERIALS AND METHODS}

Study is based on morphometric analysis of 402 cranial skeleton elements: 190 skulls and 212 mandibles (Tabs 1, 2). The material, housed in the Institute of Systematics and Evolution of Animals, Polish Academy of Sciences in Kraków (ISEA, PAS), came from 17 thanatocoenoses, dated with ${ }^{14} \mathrm{C}$ AMS method (Tab. 3).

Time scale includes the period from $5710 \mathrm{BC}-5570 \mathrm{BC}(6725 \pm 50$ bp before calibration) to the recent time.${ }^{14} \mathrm{C}$ dates, calibrated in OxCal programme, are given with $60 \%$ probability (standard in zoological analysis) (Walanus \& Goslar, 2004).

For statistics, the following computer programmes were used: Microsoft Excel, Past, Statistica 6.0, and Canoco 4.52. For Discriminant Analysis (DA) and for making diagrams of changes morphological parameters during the Middle and Late Holocene, samples of unless 9 cranial skeleton elements were used.

\section{Abbreviations}

Analysed morphological parameters:

Skull dimensions: GLS - greatest lenght of skull; CLS - condylobasal lenght of skull; $\mathrm{PM}_{1}$ palate width at $\mathrm{M}_{1}$; MSW - maximum skull width; MZB - maximum zygomatic breadth; PSW posterior skull width; COW - condylus occipitalis width; TPL - total palate lenght; $\mathrm{L}\left(\mathrm{C}-\mathrm{P}_{3}\right)$ - canine to third premolar length; LRT - toothrow lenght; $\mathrm{L}\left(\mathrm{P}_{4}-\mathrm{M}_{3}\right)-\mathrm{P}_{4}$ to $\mathrm{M}_{3}$ lenght; $\mathrm{L}\left(\mathrm{M}_{1}-\mathrm{M}_{3}\right)-\mathrm{M}_{1}$ to $\mathrm{M}_{3}$ lenght; $\mathrm{PC}$ - palatal width at canine $\left(\mathrm{C}_{1}\right)$; FCW - fossa condylaris width; $\mathrm{L}\left(\mathrm{P}_{1}-\mathrm{P}_{3}\right)-\mathrm{P}_{1}$ to $\mathrm{P}_{3}$ lenght; Mandible dimensions: MDL - mandible lenght; MBTR - mandible toothrow lenght; LPA lenght of processus angularis; $\mathrm{HCP}$ - height of coronoid process; $\mathrm{HP}_{4}$ - height of mandible at $\mathrm{P}_{4} ; \mathrm{HM}_{3}$ - height of mandible at $\mathrm{M}_{3}$.

Table 1

Mean, minimum and maximum values of skull dimensions of Bechstein's bat (Myotis bechsteinii) from thanatocoenoses dated with ${ }^{14} \mathrm{C}$ AMS method.

\begin{tabular}{|c|c|c|c|c|c|c|c|c|c|c|c|c|c|c|c|c|c|}
\hline \multirow[b]{2}{*}{${ }^{14} \mathrm{C} \mathrm{bp}$} & \multirow[b]{2}{*}{$\mathrm{N}$} & \multirow[b]{2}{*}{ Value } & \multicolumn{15}{|c|}{ Skull measurements $[\mathrm{cm}]$} \\
\hline & & & GLS & CLS & $\mathrm{PM}_{1}$ & MSW & MZB & PSW & $\mathrm{COW}$ & TPL & C- $\mathrm{P}_{3}$ & LTR & $\begin{array}{l}\mathrm{P}_{4^{-}} \\
\mathrm{M}_{3}\end{array}$ & $\begin{array}{l}\mathrm{M}_{1}- \\
\mathrm{M}_{3}\end{array}$ & $\mathrm{PC}$ & $\mathrm{FCW}$ & $\begin{array}{l}\mathrm{P}_{1^{-}} \\
\mathrm{P}_{3} \\
\end{array}$ \\
\hline \multirow[t]{3}{*}{6725} & \multirow[t]{3}{*}{14} & mean & 17.27 & 16.31 & 5.72 & 4.22 & 11.16 & 8.40 & 4.92 & 8.05 & 2.25 & 6.74 & 4.69 & 3.75 & 3.76 & 3.91 & 1.23 \\
\hline & & minimum & 17.16 & 16.22 & 5.59 & 4.17 & 11.12 & 8.30 & 4.52 & 7.88 & 2.17 & 6.40 & 4.63 & 3.54 & 3.60 & 3.68 & 1.01 \\
\hline & & maximum & 17.42 & 16.43 & 6.20 & 4.30 & 1.22 & 8.51 & 5.31 & 8.25 & 2.38 & 6.94 & 4.80 & 3.91 & 4.00 & 4.01 & 1.41 \\
\hline \multirow[t]{3}{*}{5990} & \multirow[t]{3}{*}{10} & mean & 17.53 & 16.46 & 5.77 & 4.30 & 10.88 & 8.28 & 4.93 & 8.89 & 2.34 & 6.69 & 4.71 & 3.62 & 3.77 & 4.13 & 1.32 \\
\hline & & minimum & 17.23 & 16.28 & 5.66 & 4.21 & 10.58 & 8.18 & 4.70 & 7.98 & 2.22 & 6.53 & 4.67 & 3.48 & 3.54 & 3.88 & 1.11 \\
\hline & & maximum & 17.83 & 16.67 & 5.84 & 4.40 & 11.23 & 8.46 & 5.22 & 9.92 & 2.40 & 7.00 & 4.73 & 3.83 & 3.97 & 4.27 & 1.42 \\
\hline \multirow[t]{3}{*}{4925} & \multirow[t]{3}{*}{25} & mean & 17.43 & 16.48 & 5.67 & 4.43 & 10.91 & 8.31 & 4.94 & 8.52 & 2.35 & 6.80 & 4.73 & 3.84 & 4.02 & 4.03 & 1.39 \\
\hline & & minimum & 17.19 & 16.24 & 5.49 & 4.19 & 10.59 & 8.16 & 4.72 & 7.68 & 2.21 & 6.48 & 4.52 & 3.64 & 3.77 & 3.79 & 1.10 \\
\hline & & maximum & 17.99 & 16.87 & 6.00 & 4.97 & 11.20 & 8.52 & 5.17 & 8.99 & 2.47 & 7.21 & 4.92 & 4.74 & 4.22 & 4.38 & 1.68 \\
\hline
\end{tabular}


Table 1 (continued)

\begin{tabular}{|c|c|c|c|c|c|c|c|c|c|c|c|c|c|c|c|c|c|}
\hline \multirow[b]{2}{*}{${ }^{14} \mathrm{C} \mathrm{bp}$} & \multirow[b]{2}{*}{$\mathrm{N}$} & \multirow[b]{2}{*}{ Value } & \multicolumn{15}{|c|}{ Skull measurements [cm] } \\
\hline & & & GLS & CLS & $\mathrm{PM}_{1}$ & MSW & MZB & PSW & COW & TPL & C-P & LTR & $\begin{array}{l}\mathrm{P}_{4^{-}} \\
\mathrm{M}_{3} \\
\end{array}$ & \begin{tabular}{|l|}
$\mathrm{M}_{1^{-}}$ \\
$\mathrm{M}_{3}$ \\
\end{tabular} & $\mathrm{PC}$ & FCW & $\begin{array}{l}\mathrm{P}_{1^{-}} \\
\mathrm{P}_{3} \\
\end{array}$ \\
\hline \multirow[t]{3}{*}{4345} & \multirow[t]{3}{*}{5} & mean & 17.42 & 16.67 & 5.82 & 4.80 & 10.87 & 8.28 & 4.78 & 8.70 & 2.37 & 7.05 & 4.64 & 3.91 & 3.80 & 4.18 & 1.49 \\
\hline & & minimum & 17.39 & 16.44 & 5.51 & 4.50 & 10.71 & 8.19 & 4.67 & 8.62 & 2.02 & 6.72 & 4.52 & 3.86 & 3.77 & 4.06 & 1.42 \\
\hline & & maximum & 17.46 & 16.86 & 6.01 & 5.00 & 11.00 & 8.41 & 4.83 & 8.75 & 2.71 & 7.33 & 4.78 & 4.01 & 3.86 & 4.29 & 1.58 \\
\hline \multirow[t]{3}{*}{3725} & \multirow[t]{3}{*}{12} & mean & 17.40 & 16.51 & 5.71 & 4.48 & 10.73 & 8.33 & 4.90 & 9.44 & 2.39 & 7.04 & 4.66 & 3.73 & 3.79 & 4.10 & 1.45 \\
\hline & & minimum & 17.12 & 16.41 & 5.56 & 4.30 & 10.50 & 8.23 & 4.72 & 8.77 & 2.30 & 6.81 & 4.42 & 3.51 & 3.53 & 3.80 & 1.31 \\
\hline & & maximum & 17.81 & 16.62 & 5.82 & 4.68 & 11.00 & 8.46 & 5.01 & 10.09 & 2.49 & 7.21 & 4.85 & 3.88 & 4.66 & 4.23 & 1.62 \\
\hline \multirow[t]{3}{*}{3545} & \multirow[t]{3}{*}{12} & mean & 17.39 & 16.91 & 5.82 & 4.32 & 10.60 & 7.97 & 4.96 & 9.77 & 2.43 & 6.98 & 4.60 & 3.69 & 4.00 & 4.24 & 1.84 \\
\hline & & minimum & 17.21 & 16.60 & 5.60 & 4.01 & 10.26 & 7.60 & 4.74 & 8.98 & 2.23 & 6.70 & 4.41 & 3.63 & 3.67 & 4.00 & 1.40 \\
\hline & & ximum & 17.61 & 17.19 & 5.92 & 4.90 & 10.90 & 8.33 & 5.21 & 10.31 & 2.58 & 7.25 & 5.01 & 3.79 & 4.23 & 4.36 & 2.24 \\
\hline \multirow[t]{3}{*}{2890} & \multirow[t]{3}{*}{20} & mean & 17.70 & 16.57 & 5.80 & 4.54 & 10.88 & 8.49 & 4.91 & 9.47 & 2.47 & 6.94 & 4.45 & 3.46 & 3.66 & 3.95 & 1.40 \\
\hline & & minimum & 17.46 & 16.39 & 5.67 & 4.29 & 10.70 & 8.21 & 4.70 & 8.72 & 2.24 & 6.52 & 4.23 & 3.22 & 3.50 & 3.79 & 1.33 \\
\hline & & ximum & 17.90 & 16.73 & 5.93 & 5.00 & 11.04 & 8.83 & 5.10 & 10.02 & 2.74 & 7.45 & 4.73 & 3.83 & 3.78 & 4.28 & 1.48 \\
\hline \multirow[t]{3}{*}{2120} & \multirow[t]{3}{*}{24} & mean & 17.58 & 16.40 & 5.67 & 4.39 & 10.78 & 8.34 & 4.85 & 9.07 & 2.42 & 6.69 & 4.26 & 3.32 & 3.55 & 3.86 & 1.35 \\
\hline & & nimum & 17.45 & 16.22 & 5.59 & 4.29 & 10.63 & 8.21 & 4.70 & 8.52 & 2.21 & 6.45 & 3.82 & 3.19 & 3.40 & 3.62 & 1.27 \\
\hline & & maximum & 17.77 & 16.66 & 5.77 & 4.63 & 11.00 & 8.44 & 5.02 & 9.56 & 2.68 & 6.88 & 4.58 & 3.45 & 3.66 & 4.18 & 1.40 \\
\hline \multirow[t]{3}{*}{1490} & \multirow[t]{3}{*}{6} & mean & 17.36 & 16.33 & 5.76 & 4.32 & 10.95 & 8.30 & 5.20 & 8.23 & 2.24 & 6.92 & 4.68 & 4.43 & 3.80 & 3.94 & 1.19 \\
\hline & & inimum & 17.29 & 16.25 & 5.66 & 4.20 & 10.80 & 8.21 & 5.12 & 8.17 & 2.16 & 6.84 & 4.65 & 3.70 & 3.76 & 3.80 & 1.06 \\
\hline & & maximum & 17.42 & 16.41 & 5.82 & 4.45 & 11.11 & 8.41 & 5.30 & 8.30 & 2.32 & 7.00 & 4.71 & 7.79 & 3.82 & 4.02 & 1.37 \\
\hline \multirow[t]{3}{*}{1470} & \multirow[t]{3}{*}{15} & ean & 17.44 & 16.35 & 5.84 & 4.29 & 11.00 & 8.35 & 5.00 & 8.18 & 2.21 & 6.89 & 4.79 & 3.80 & 3.80 & 4.00 & 1.08 \\
\hline & & minimum & 17.29 & 16.20 & 5.31 & 4.20 & 10.75 & 8.21 & 4.82 & 7.96 & 2.15 & 6.80 & 4.62 & 3.70 & 3.76 & 3.90 & 1.00 \\
\hline & & maximum & 17.67 & 16.42 & 6.20 & 4.40 & 11.22 & 8.41 & 5.22 & 8.30 & 2.31 & 7.00 & 6.66 & 3.89 & 3.83 & 4.12 & 1.18 \\
\hline \multirow[t]{3}{*}{1320} & \multirow[t]{3}{*}{21} & mean & 17.55 & 16.54 & 5.83 & 4.41 & 10.84 & 8.47 & 4.97 & 8.74 & 2.27 & 6.79 & 4.52 & 3.68 & 3.78 & 4.00 & 1.21 \\
\hline & & minimum & 17.31 & 16.35 & 5.66 & 4.30 & 10.52 & 8.31 & 4.78 & 8.18 & 2.18 & 6.53 & 4.25 & 3.34 & 3.61 & 3.76 & 1.00 \\
\hline & & maximum & 17.88 & 16.73 & 6.12 & 4.63 & 11.22 & 8.83 & 5.22 & 9.94 & 2.41 & 7.00 & 4.78 & 3.86 & 3.89 & 4.22 & 1.40 \\
\hline \multirow[t]{3}{*}{595} & \multirow[t]{3}{*}{14} & mean & 17.73 & 16.60 & 5.74 & 4.42 & 10.75 & 8.05 & 5.28 & 8.30 & 2.30 & 6.91 & 4.76 & 3.66 & 3.85 & 4.06 & 1.36 \\
\hline & & minimum & 17.49 & 16.33 & 5.00 & 4.27 & 10.56 & 7.69 & 5.11 & 8.11 & 2.18 & 6.74 & 4.48 & 3.45 & 3.61 & 3.79 & 1.29 \\
\hline & & maximum & 18.12 & 16.77 & 5.99 & 4.66 & 10.99 & 8.43 & 5.40 & 8.62 & 2.42 & 7.04 & 6.60 & 3.88 & 4.01 & 4.35 & 1.50 \\
\hline \multirow[t]{3}{*}{1} & \multirow[t]{3}{*}{12} & mean & 17.70 & 16.95 & 5.71 & 4.43 & 10.81 & 7.95 & 5.45 & 9.70 & 2.32 & 6.95 & 4.63 & 3.76 & 4.02 & 4.33 & 2.17 \\
\hline & & minimum & 17.17 & 16.64 & 5.01 & 4.17 & 10.47 & 7.63 & 5.12 & 8.87 & 2.23 & 6.80 & 4.48 & 3.66 & 3.88 & 4.19 & 1.93 \\
\hline & & maximum & 18.09 & 17.28 & 5.99 & 4.62 & 11.02 & 8.40 & 5.79 & 10.22 & 2.40 & 7.24 & 4.87 & 3.93 & 4.13 & 4.49 & 2.36 \\
\hline$\Sigma$ & 190 & & & & & & & & & & & & & & & & \\
\hline
\end{tabular}


Table 2

Mean, minimum and maximum values of mandible dimensions of Bechstein's bat (Myotis bechsteinii) from thanatocoenoses dated with ${ }^{14} \mathrm{C}$ AMS method.

\begin{tabular}{|c|c|c|c|c|c|c|c|c|}
\hline \multirow{2}{*}{${ }^{14} \mathrm{C}$ bp } & \multirow{2}{*}{$\mathrm{N}$} & \multirow{2}{*}{ Value } & \multicolumn{6}{|c|}{ Mandible measurements [cm] } \\
\hline & & & MDL & MBTR & LPA & $\mathrm{HCP}$ & $\mathrm{HP}_{4}$ & $\mathrm{HM}_{3}$ \\
\hline \multirow[t]{3}{*}{6725} & \multirow[t]{3}{*}{19} & mean & 12.72 & 7.43 & 3.92 & 4.00 & 1.50 & 1.58 \\
\hline & & minimum & 12.16 & 6.36 & 3.37 & 3.73 & 1.32 & 1.43 \\
\hline & & maximum & 13.37 & 7.90 & 4.55 & 4.25 & 1.74 & 1.78 \\
\hline \multirow[t]{3}{*}{5990} & \multirow[t]{3}{*}{9} & mean & 12.99 & 7.43 & 3.93 & 4.04 & 1.46 & 1.62 \\
\hline & & minimum & 12.91 & 6.93 & 3.46 & 3.78 & 1.35 & 1.40 \\
\hline & & maximum & 13.09 & 7.78 & 4.27 & 4.17 & 1.55 & 1.72 \\
\hline \multirow[t]{3}{*}{5225} & \multirow[t]{3}{*}{20} & mean & 12.87 & 7.39 & 4.05 & 4.01 & 1.55 & 1.65 \\
\hline & & minimum & 12.45 & 6.98 & 3.52 & 3.42 & 1.42 & 1.52 \\
\hline & & maximum & 13.19 & 7.72 & 4.34 & 4.34 & 1.68 & 1.79 \\
\hline \multirow[t]{3}{*}{4345} & \multirow[t]{3}{*}{20} & mean & 12.61 & 7.33 & 3.96 & 3.93 & 1.53 & 1.59 \\
\hline & & minimum & 12.22 & 6.99 & 3.52 & 3.44 & 1.35 & 1.36 \\
\hline & & maximum & 13.01 & 7.54 & 4.27 & 4.35 & 1.68 & 1.79 \\
\hline \multirow[t]{3}{*}{4110} & \multirow[t]{3}{*}{12} & mean & 12.47 & 7.24 & 3.76 & 3.84 & 1.41 & 1.53 \\
\hline & & minimum & 11.93 & 7.00 & 3.50 & 3.33 & 1.31 & 1.36 \\
\hline & & maximum & 12.99 & 7.50 & 4.24 & 4.35 & 1.56 & 1.64 \\
\hline \multirow[t]{3}{*}{3905} & \multirow[t]{3}{*}{9} & mean & 13.07 & 7.44 & 4.06 & 4.01 & 1.53 & 1.61 \\
\hline & & minimum & 12.72 & 7.34 & 3.77 & 3.80 & 1.45 & 1.56 \\
\hline & & maximum & 13.42 & 7.64 & 4.29 & 4.34 & 1.66 & 1.70 \\
\hline \multirow[t]{3}{*}{3545} & \multirow[t]{3}{*}{26} & mean & 12.79 & 7.58 & 3.80 & 3.78 & 1.61 & 1.64 \\
\hline & & minimum & 12.60 & 7.29 & 3.60 & 3.65 & 1.49 & 1.58 \\
\hline & & maximum & 13.00 & 7.89 & 4.01 & 4.01 & 1.68 & 1.70 \\
\hline \multirow[t]{3}{*}{3535} & \multirow[t]{3}{*}{20} & mean & 12.95 & 7.60 & 3.91 & 3.93 & 1.61 & 1.68 \\
\hline & & minimum & 12.80 & 7.29 & 3.68 & 3.65 & 1.57 & 1.62 \\
\hline & & maximum & 13.09 & 7.82 & 4.11 & 4.12 & 1.64 & 1.71 \\
\hline \multirow[t]{3}{*}{2890} & \multirow[t]{3}{*}{24} & mean & 12.76 & 7.44 & 3.83 & 3.85 & 1.63 & 1.66 \\
\hline & & minimum & 12.36 & 7.23 & 3.51 & 3.45 & 1.43 & 1.59 \\
\hline & & maximum & 13.20 & 7.79 & 4.11 & 4.11 & 1.83 & 1.77 \\
\hline \multirow[t]{3}{*}{2415} & \multirow[t]{3}{*}{21} & mean & 12.76 & 7.44 & 3.72 & 3.88 & 1.61 & 1.66 \\
\hline & & minimum & 12.47 & 6.90 & 3.51 & 3.58 & 1.50 & 1.59 \\
\hline & & maximum & 13.22 & 7.72 & 4.00 & 4.11 & 1.72 & 1.71 \\
\hline \multirow[t]{3}{*}{1470} & \multirow[t]{3}{*}{5} & mean & 12.81 & 7.56 & 4.27 & 4.15 & 1.52 & 1.50 \\
\hline & & minimum & 12.69 & 7.49 & 4.12 & 4.01 & 1.44 & 1.47 \\
\hline & & maximum & 12.99 & 7.62 & 4.32 & 4.30 & 1.58 & 1.53 \\
\hline
\end{tabular}


Table 2 (continued)

\begin{tabular}{|c|c|c|c|c|c|c|c|c|}
\hline \multirow{2}{*}{${ }^{14} \mathrm{C}$ bp } & \multirow[b]{2}{*}{$\mathrm{N}$} & \multirow{2}{*}{ Value } & \multicolumn{6}{|c|}{ Mandible measurements [cm] } \\
\hline & & & MDL & MBTR & LPA & HCP & $\mathrm{HP}_{4}$ & $\mathrm{HM}_{3}$ \\
\hline \multirow[t]{3}{*}{1320} & \multirow[t]{3}{*}{3} & mean & 12.78 & 7.61 & 4.35 & 4.26 & 1.56 & 1.54 \\
\hline & & minimum & 12.77 & 7.60 & 4.33 & 4.20 & 1.55 & 1.53 \\
\hline & & maximum & 12.79 & 7.63 & 4.37 & 4.30 & 1.57 & 1.54 \\
\hline \multirow[t]{3}{*}{595} & \multirow[t]{3}{*}{6} & mean & 12.73 & 7.58 & 4.28 & 4.21 & 1.57 & 1.52 \\
\hline & & minimum & 12.70 & 7.52 & 4.20 & 4.19 & 1.55 & 1.50 \\
\hline & & maximum & 12.77 & 7.62 & 4.36 & 4.24 & 1.60 & 1.53 \\
\hline \multirow[t]{3}{*}{1} & \multirow[t]{3}{*}{18} & mean & 12.80 & 7.52 & 4.29 & 4.38 & 1.57 & 1.52 \\
\hline & & minimum & 12.69 & 7.35 & 4.20 & 4.30 & 1.51 & 1.48 \\
\hline & & maximum & 12.93 & 7.81 & 4.41 & 4.54 & 1.60 & 1.55 \\
\hline$\Sigma$ & 212 & & & & & & & \\
\hline
\end{tabular}

Table 3

Thanatocoenoses dated with ${ }^{14} \mathrm{C}$ AMS method (1-3, 5-7 and 10 from Kraków-Częstochowa Upland; 4, 8, 11 and 13-17 from Tatra Mts.; 9 from Świętokrzyskie Mts.; 12 from Sudety Mts.; 18 - recent specimens from uplands of southern Poland).

\begin{tabular}{|c|c|c|c|}
\hline Nr. & Thanatocoenoses & ${ }^{14} \mathrm{C}$ bp AMS & $\begin{array}{l}{ }^{14} \mathrm{C} \text { BP AMS } \\
\text { Calibrated date }\end{array}$ \\
\hline 1 & Cave Na Świniuszce & $6725 \pm 50 \mathrm{BP}$ & $5710 \mathrm{BC}-5570 \mathrm{BC}$ \\
\hline 2 & Cave Studnisko II & $5990 \pm 40 \mathrm{BP}$ & $4940 \mathrm{BC}-4800 \mathrm{BC}$ \\
\hline 3 & Cave Maurycego & $5225 \pm 45 \mathrm{BP}$ & $4150 \mathrm{BC}-3960 \mathrm{BC}$ \\
\hline 4 & Cave Bandzioch Kominiarski & $4925 \pm 35 \mathrm{BP}$ & $3760 \mathrm{BC}-3650 \mathrm{BC}$ \\
\hline 5 & Cave Studnisko I & $4345 \pm 45 \mathrm{BP}$ & $3020 \mathrm{BC}-2900 \mathrm{BC}$ \\
\hline 6 & Cave Krysztatowa & $4110 \pm 45 \mathrm{BP}$ & $2860 \mathrm{BC}-2580 \mathrm{BC}$ \\
\hline 7 & Cave Małotowa & $3905 \pm 45 \mathrm{BP}$ & $2470 \mathrm{BC}-2340 \mathrm{BC}$ \\
\hline 8 & Cave Miętusia Wyżnia & $3725 \pm 30 \mathrm{BP}$ & $2200 \mathrm{BC}-2040 \mathrm{BC}$ \\
\hline 9 & Cave Zbójecka w Łagowie & $3545 \pm 35 \mathrm{BP}$ & $1940 \mathrm{BC}-1780 \mathrm{BC}$ \\
\hline 10 & Cave Na Tomaszówkach Dln. & $3535 \pm 35 \mathrm{BP}$ & $1930 \mathrm{BC}-1770 \mathrm{BC}$ \\
\hline 11 & Cave Wysoka Za Siedmioma Progami & $2890 \pm 30 \mathrm{BP}$ & $1120 \mathrm{BC}-1015 \mathrm{BC}$ \\
\hline 12 & Cave Niedźwiedzia w Kletnie & $2415 \pm 30 \mathrm{BP}$ & $530 \mathrm{BC}-400 \mathrm{BC}$ \\
\hline 13 & Cave Zakosista & $2120 \pm 30 \mathrm{BP}$ & $200 \mathrm{BC}-100 \mathrm{BC}$ \\
\hline 14 & Cave Szczelina Chochołowska & $1490 \pm 30 \mathrm{BP}$ & $545 \mathrm{AD}-605 \mathrm{AD}$ \\
\hline 15 & Cave Piwnica Miętusia & $1470 \pm 30 \mathrm{BP}$ & $565 \mathrm{AD}-630 \mathrm{AD}$ \\
\hline 16 & Cave Zimna & $1370 \pm 30 \mathrm{BP}$ & $640 \mathrm{AD}-670 \mathrm{AD}$ \\
\hline 17 & Cave Czarna & $595 \pm 30 \mathrm{BP}$ & $1305 \mathrm{AD}-1405 \mathrm{AD}$ \\
\hline 18 & Recent specimens & $*$ marked as 1 & \\
\hline
\end{tabular}


At each skull 15 and at each mandible six morphological parameters were taken, using Nikon MM200 measurement microscope. Each result was given with precision up to $0.001 \mathrm{~mm}$ (Tabs 1,2).

To divide Middle and Late Holocene population of M. bechsteinii into general groups, and to see eventually mistakes in description of specimens as Bechstein's bat, Principal Component Analysis (PCA) was made.

Discriminant Analysis (DA) gives an answer to which of several cranial dimensions have significant and the strongest influence on differenciated investigated populations. Than changes of each morpholgical parameter significantly discriminating these groups was examined. 28 environmental factors were chosen to investigate their correlation with morphological parameters. Those are: age of sample, temperature of January, general climate characteristic, a.s.l. hight, frequency of Bechstein's bat, frequency of Natterer's bat, percentage of pollen of: fir, maple, hornbeam, beech, ash, spruce, pine, oak, willow, lime, elm, sum of herb pollen, etc. (Tab. 7).

To see which of environmental variables (Tab. 4) have significant influence to morphology and if it correlates with morphology positively or negatively, Redundancy Analysis (RDA) with gradient selection was made (Lepš \& Šmilauer, 1999; Ter Braak \& Prentice, 1988). To make results clearer, time limits are given separately for Holocene: Atlantic $\left(8200-5115{ }^{14} \mathrm{C}\right.$ BP), Subboreal $\left(5115-2800{ }^{14} \mathrm{C}\right.$ BP), and Subatlantic period ( $2800{ }^{14} \mathrm{C}$ BP till now). In Redundancy Analysis (RDA) lenght and direction of the arrows say about strenght and character of correlation with morphological parameters (the longer arrow $=$ the stronger influence on environmental parameter to morphology).

\section{RESULTS}

For skulls as well as for mandibles percentage of variance for the first with the second canonical axis in PCA does not exceed 50\%: there is no doubt that all specimens belong to the species $M$. bechsteinii (Tab. 5). Results of PCA are given on figures 1 and 2. Differently marked lines connect extreme points of each group, dated with ${ }^{14} \mathrm{C}$ method. Influence of sexual dimorphism to differences between populations of Bechstein's bat from radiocarbon dated thanatocoenoses are excluded by researching recent population of the species. Principal Component Analysis did not show signifficant differences between males and females (variance $=38 \%$ ).

Discriminant analysis shows morphological parameters significantly differenciating examined populations. In skull morphology those are: $\mathrm{P}^{1}-\mathrm{P}^{3}$ length $\left[\mathrm{L}\left(\mathrm{P}^{1}-\mathrm{P}^{3}\right)\right]$, lenght from the first to the third molar $\left[\mathrm{L}\left(\mathrm{M}_{1}-\mathrm{M}_{3}\right)\right]$, maximum zygomatic breadth (MZB), total palate lenght (TPL), condylus occipitalis width (COW), posterior skull width (PSW), greatest lenght of skull (GLS), toothrow lenght (LTR), palatal width at canine $\left(\mathrm{C}^{1}\right)\left[\mathrm{L}\left(\mathrm{C}^{1}-\mathrm{C}^{1}\right)\right]$, canine to third premolar length $\left[\mathrm{L}\left(\mathrm{C}-\mathrm{P}^{3}\right)\right]$, condylobasal of skull (CLS), maximum skull width (MSW), fossa condylaris width (FCW), $\mathrm{P}^{4}$ to $\mathrm{M}^{3}$ length $\left[\mathrm{L}\left(\mathrm{P}^{4}-\mathrm{M}^{3}\right)\right]$ and in mandibles: height of coronoid process ( $\mathrm{HCP})$, height of mandible at third molar $\left(\mathrm{HM}_{3}\right)$, height of mandible at fourth premolar $\left(\mathrm{HP}_{4}\right)$, lenght of processus angularis (LPA), mandible lenght (MDL) and mandible toothrow length (MBTR) (Tab. 7). 
MICROEVOLUTION OF BECHSTEIN'S BAT IN THE HOLOCENE OF SOUTHERN POLAND 249

\begin{tabular}{|c|c|c|c|c|c|c|c|c|c|c|c|c|c|c|c|c|c|c|}
\hline 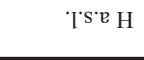 & 号 & $\stackrel{0}{m}$ & 吕 & 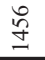 & $\stackrel{2}{2}$ & $\begin{array}{l}0 \\
\text { J } \\
\end{array}$ & $\begin{array}{l}+ \\
\infty \\
ల\end{array}$ & 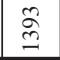 & $\frac{a}{m}$ & $\begin{array}{l}\approx \\
q \\
q\end{array}$ & 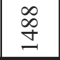 & $\overbrace{\infty}$ & 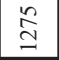 & $\overline{\tilde{s}}$ & مే & $\stackrel{\overbrace{}}{\Xi}$ & 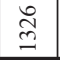 & $\underset{\sim}{\infty}$ \\
\hline$\left[\supset_{0}\right] \kappa_{\mathrm{L}}$ & 盀 & วั & Na & $\stackrel{\circ}{r}$ & $\infty$ & $\infty$ & $\infty$ & $\stackrel{q}{\dot{q}}$ & $\mid \begin{array}{l}n \\
\infty \\
\infty\end{array}$ & $\infty$ & $\stackrel{\rho}{r}$ & $\stackrel{+}{\dot{m}}$ & 官 & f & $\stackrel{n}{i}$ & $\tilde{A}$ & 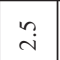 & $\stackrel{\circ}{i}$ \\
\hline spoo ${ }_{\text {SIH }}$ & 은 & $\circ$ & - & - & - & 0 & $\circ$ & 0 & 0 & 0 & 0 & -7 & - & 0 & 0 & -7 & -7 & - \\
\hline spoogl- ${ }^{-}$d & $\begin{array}{l}\text { ठ̊ } \\
\vdots \\
0\end{array}$ & 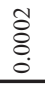 & $\begin{array}{l}\text { ¿े } \\
\vdots \\
\end{array}$ & $\begin{array}{l}\text { ڤ. } \\
\vdots \\
0\end{array}$ & 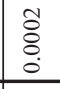 & \begin{tabular}{|l} 
\\
0 \\
0 \\
0 \\
\end{tabular} & \begin{tabular}{|l}
$\bar{\Xi}$ \\
$\dot{0}$ \\
\end{tabular} & \begin{tabular}{|l|}
$\bar{\Xi}$ \\
0 \\
\end{tabular} & \begin{tabular}{|l|}
0 \\
$\vdots$ \\
0 \\
0 \\
\end{tabular} & \begin{tabular}{|l|} 
\\
0 \\
0 \\
0 \\
\end{tabular} & 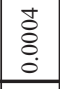 & \begin{tabular}{|c|}
0 \\
$\vdots$ \\
0 \\
0
\end{tabular} & \begin{tabular}{|l|}
\multirow{0}{0}{} \\
0 \\
0 \\
\end{tabular} & \begin{tabular}{|l} 
\\
\\
0 \\
0 \\
\end{tabular} & \begin{tabular}{|l} 
\\
0 \\
0 \\
0
\end{tabular} & $\begin{array}{l} \\
\vdots \\
0 \\
0 \\
\end{array}$ & $\begin{array}{l}\infty \\
\vdots \\
0 \\
0\end{array}$ & $\begin{array}{l}\bar{\Xi} \\
\overline{0} \\
\end{array}$ \\
\hline $\mathrm{NAW} \mathrm{I}_{\mathrm{B}}$ & 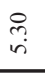 & $\begin{array}{r}\vec{J} \\
\text { in }\end{array}$ & 灾 & $\stackrel{\circ}{\circ}$ & సু & $\begin{array}{l}n \\
\text { in } \\
\text { in } \\
\end{array}$ & $\begin{array}{l}\hat{i} \\
i\end{array}$ & $\stackrel{8}{-}$ & 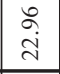 & \begin{tabular}{|l}
$\infty$ \\
$\alpha$ \\
$\alpha$
\end{tabular} & 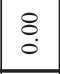 & \begin{tabular}{|l}
0 \\
$\infty$ \\
$\stackrel{0}{0}$ \\
\end{tabular} & 8 & 8 & oे & $\begin{array}{l}8 \\
0 \\
0\end{array}$ & $\stackrel{+}{+}$ & 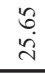 \\
\hline ggW & $\begin{array}{l}\text { 足 } \\
\text { id }\end{array}$ & 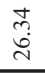 & 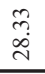 & $\stackrel{\circ}{=}$ & $\begin{array}{c}\tilde{y} \\
\infty \\
\infty\end{array}$ & $\stackrel{\bar{f}}{\bar{F}}$ & \begin{tabular}{|l}
5 \\
+ \\
\end{tabular} & 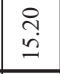 & \begin{tabular}{|l}
3 \\
$\dot{a}$ \\
$i$
\end{tabular} & \begin{tabular}{|c} 
fo \\
in \\
nn
\end{tabular} & \begin{tabular}{|}
$\sigma$ \\
$\tilde{j}$ \\
\end{tabular} & 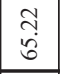 & \begin{tabular}{|l}
$\infty$ \\
\hdashline \\
$\dot{\sigma}$ \\
\end{tabular} & $\begin{array}{l}8 \\
\dot{1} \\
\end{array}$ & $\begin{array}{l}f \\
\text { in } \\
\end{array}$ & 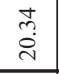 & $\begin{array}{l}\dot{\sigma} \\
\dot{\sigma}\end{array}$ & $\overbrace{0}^{\infty}$ \\
\hline әдвш! & - & - & - & 4 & a & $n$ & a & $a$ & $a$ & 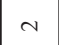 & 4 & $m$ & $m$ & $m$ & m & $m$ & $m$ & $\theta$ \\
\hline sq.ə̈H & $\begin{array}{l}8 \\
\stackrel{0}{0}\end{array}$ & $\begin{array}{l}\stackrel{8}{\circ} \\
\end{array}$ & $\begin{array}{l}8 \\
\dot{\leftrightarrow} \\
\end{array}$ & $\begin{array}{l}\stackrel{8}{\circ} \\
\end{array}$ & \begin{tabular}{|l}
$\stackrel{8}{0}$ \\
\\
\end{tabular} & $\begin{array}{l}\stackrel{8}{\circ} \\
\end{array}$ & \begin{tabular}{|l}
8 \\
0 \\
0 \\
\end{tabular} & \begin{tabular}{|l}
$\stackrel{8}{0}$ \\
\\
\end{tabular} & 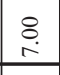 & $\begin{array}{r}8 \\
\dot{a} \\
\end{array}$ & $\underset{8}{\stackrel{2}{1}}$ & 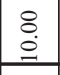 & \begin{tabular}{|l}
8 \\
$\stackrel{0}{0}$ \\
\end{tabular} & \begin{tabular}{|r}
8 \\
$\dot{1}$ \\
\end{tabular} & $\begin{array}{r}8 \\
0 \\
0\end{array}$ & $\begin{array}{l}8 \\
\stackrel{+}{1} \\
\end{array}$ & $\begin{array}{l}8 \\
0 \\
0 \\
\end{array}$ & $\begin{array}{l}8 \\
\stackrel{9}{+} \\
\end{array}$ \\
\hline${ }^{p y d \kappa_{L}}$ & ণั่ & त్రి & $\stackrel{8}{0}$ & ণิ่ & ָุ & वृ. & กิ & वृ & वृ & 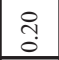 & $\begin{array}{l} \\
\text { yु } \\
\end{array}$ & $\begin{array}{l}\text { ị } \\
\\
\end{array}$ & \begin{tabular}{|c|}
\multirow{1}{*}{} \\
\end{tabular} & ণั & ণ্ণ & ণ্ & ֻृ & त् \\
\hline әвәэво & $\stackrel{8}{i}$ & $\stackrel{8}{8}$ & $\stackrel{8}{8}$ & $\stackrel{8}{i}$ & $\underset{8}{8}$ & $\begin{array}{l}8 \\
i \\
i\end{array}$ & $\underset{i}{8}$ & $\underset{8}{8}$ & $\stackrel{8}{.}$ & $\begin{array}{l}8 \\
i \\
i\end{array}$ & $\underset{8}{8}$ & $\begin{array}{c}8 \\
i \\
i\end{array}$ & $\underset{8}{8}$ & 8 & $\stackrel{8}{i}$ & $\stackrel{8}{8}$ & $\stackrel{8}{\circ}$ & $\stackrel{8}{\circ}$ \\
\hline әвәәв.ә $\kappa_{\supset}$ & $\stackrel{8}{i}$ & $\stackrel{8}{i}$ & $\stackrel{8}{i}$ & $\stackrel{8}{i}$ & $\begin{array}{l}8 \\
i \\
i\end{array}$ & $\begin{array}{l}8 \\
\text { in }\end{array}$ & $\stackrel{8}{8}$ & 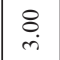 & 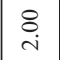 & $\begin{array}{l}8 \\
i \\
i\end{array}$ & $\underset{-}{\stackrel{1}{-}}$ & 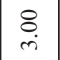 & $\begin{array}{l}8 \\
i \\
i\end{array}$ & $\underset{j}{8}$ & 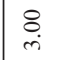 & $\underset{丶}{\stackrel{r}{r}}$ & $\begin{array}{l}8 \\
\dot{i n}\end{array}$ & $\begin{array}{l}8 \\
\stackrel{0}{0}\end{array}$ \\
\hline 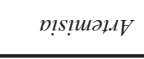 & $\stackrel{R}{0}$ & : & $\stackrel{R}{0}$ & $\stackrel{8}{-}$ & $\begin{array}{l}R \\
0 \\
0\end{array}$ & in & \begin{tabular}{|l}
8 \\
0 \\
0
\end{tabular} & $\underset{8}{\stackrel{8}{-}}$ & $\underset{+}{\stackrel{-}{+}}$ & \begin{tabular}{|l|}
2 \\
0 \\
0
\end{tabular} & $\underset{8}{\stackrel{8}{-}}$ & $\underset{-}{\stackrel{8}{-}}$ & $\underset{8}{\stackrel{8}{-}}$ & $\underset{8}{8}$ & $\stackrel{8}{-}$ & $\stackrel{8}{-}$ & $\underset{i}{\stackrel{i}{i}}$ & $\underset{\mathrm{i}}{\stackrel{\mathrm{j}}{ }}$ \\
\hline מวว!d & 8 & 8 & 8 & 8 & $\begin{array}{l}8 \\
\\
0\end{array}$ & $\underset{1}{\stackrel{8}{1}}$ & $\begin{array}{l}8 \\
8 \\
1 \\
1\end{array}$ & \begin{tabular}{|c|}
8 \\
0 \\
$i$ \\
1
\end{tabular} & $\begin{array}{l}8 \\
i \\
\text {. }\end{array}$ & \begin{tabular}{|l|} 
\\
\\
0
\end{tabular} & \begin{tabular}{|l|} 
\\
8 \\
4 \\
$n$
\end{tabular} & $\underset{1}{8}$ & \begin{tabular}{|l|} 
\\
\\
0
\end{tabular} & $\stackrel{8}{8}$ & $\stackrel{8}{\circ}$ & $\stackrel{8}{\circ}$ & $\underset{j}{8}$ & $\underset{\mathrm{i}}{8}$ \\
\hline${ }_{\text {snu! }}$ & $\begin{array}{l}8 \\
\stackrel{8}{0} \\
\end{array}$ & $\begin{array}{l}8 \\
\stackrel{m}{0}\end{array}$ & 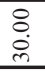 & $\begin{array}{l}8 \\
\stackrel{4}{4} \\
\end{array}$ & \begin{tabular}{|r}
8 \\
4 \\
4 \\
\end{tabular} & \begin{tabular}{|c|} 
\\
\\
0 \\
\end{tabular} & \begin{tabular}{|l}
8 \\
0 \\
4 \\
\end{tabular} & \begin{tabular}{|l|} 
\\
$\stackrel{1}{1}$ \\
\end{tabular} & \begin{tabular}{|l|} 
\\
$\dot{+}$ \\
\end{tabular} & \begin{tabular}{|r}
8 \\
6 \\
4 \\
\end{tabular} & \begin{tabular}{|c|} 
\\
\\
$\dot{i}$ \\
\end{tabular} & \begin{tabular}{|c}
8 \\
8 \\
id \\
\end{tabular} & \begin{tabular}{|r|} 
\\
8 \\
4 \\
\end{tabular} & \begin{tabular}{|l} 
\\
0 \\
0 \\
\end{tabular} & $\begin{array}{r}8 \\
\stackrel{1}{1} \\
\end{array}$ & $\begin{array}{l}8 \\
\stackrel{\tilde{~}}{ } \\
\end{array}$ & $\begin{array}{l}8 \\
8 \\
0 \\
\end{array}$ & $\begin{array}{l}8 \\
\dot{+} \\
\end{array}$ \\
\hline snuıd.tp & $\stackrel{\circ}{0}$ & : & in & $\stackrel{8}{-}$ & $\begin{array}{l}8 \\
\text { i }\end{array}$ & $\underset{+}{\stackrel{8}{+}}$ & 兽 & $\underset{\text { in }}{8}$ & $\begin{array}{l}8 \\
\text { i }\end{array}$ & 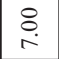 & 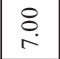 & $\underset{\sim}{\stackrel{8}{r}}$ & 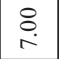 & 豙 & $\underset{\sim}{\stackrel{8}{*}}$ & $\stackrel{8}{\stackrel{1}{r}}$ & $\begin{array}{l}8 \\
i \\
\end{array}$ & $\underset{\mathrm{i}}{\stackrel{\mathrm{i}}{ }}$ \\
\hline$p ! ! ! L$ & $\underset{i}{i}$ & $\stackrel{8}{i}$ & $\stackrel{8}{i}$ & $\stackrel{8}{i}$ & $\begin{array}{l}8 \\
i \\
i\end{array}$ & $\begin{array}{l}8 \\
\\
\end{array}$ & $\begin{array}{l}8 \\
8 \\
i\end{array}$ & $\begin{array}{l}8 \\
\\
\end{array}$ & $\begin{array}{l}8 \\
i \\
i\end{array}$ & \begin{tabular}{|l|}
8 \\
$\dot{m}$
\end{tabular} & $\begin{array}{l}\underset{i}{i} \\
\text { i }\end{array}$ & $\underset{-}{\stackrel{8}{-}}$ & $\underset{-}{\stackrel{8}{*}}$ & $\stackrel{8}{8}$ & $\stackrel{8}{-}$ & $\stackrel{8}{-}$ & $\begin{array}{l}8 \\
8 \\
0\end{array}$ & ถి \\
\hline $\operatorname{sn} . .20 n \tilde{O}$ & $\stackrel{8}{8}$ & $\stackrel{8}{\circ}$ & $\stackrel{8}{\circ}$ & $\stackrel{8}{i}$ & \begin{tabular}{|l|} 
\\
\\
0
\end{tabular} & $\begin{array}{l} \\
\\
\end{array}$ & \begin{tabular}{|l} 
\\
\\
0 \\
0
\end{tabular} & $\underset{8}{8}$ & \begin{tabular}{|l|} 
\\
\\
0
\end{tabular} & \begin{tabular}{|l|} 
\\
\\
0
\end{tabular} & \begin{tabular}{|l|} 
\\
\\
\end{tabular} & $\underset{ }{\stackrel{1}{r}}$ & \begin{tabular}{|l|} 
\\
\\
\end{tabular} & $\underset{8}{8}$ & $\underset{1}{8}$ & 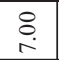 & $\begin{array}{l}8 \\
8 \\
i\end{array}$ & $\underset{8}{8}$ \\
\hline $\operatorname{snu}_{I \Omega}$ & 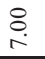 & $\stackrel{8}{8}$ & $\begin{array}{l}8 \\
\dot{n}\end{array}$ & $\stackrel{8}{\stackrel{8}{0}}$ & \begin{tabular}{|l}
8 \\
\end{tabular} & \begin{tabular}{|l}
8 \\
in \\
\end{tabular} & $\begin{array}{l}8 \\
i \\
\end{array}$ & 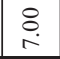 & $\begin{array}{l}\underset{\text { O }}{\mathrm{i}} \\
\mathrm{i}\end{array}$ & \begin{tabular}{|l|} 
\\
\\
\end{tabular} & \begin{tabular}{|l|}
8 \\
$i$ \\
\end{tabular} & $\begin{array}{l}\underset{i}{2} \\
\mathrm{i}\end{array}$ & \begin{tabular}{|l|} 
\\
\\
\end{tabular} & $\begin{array}{l}8 \\
\text { i } \\
\end{array}$ & $\begin{array}{l}8 \\
i \\
\end{array}$ & $\begin{array}{l}\underset{\mathrm{i}}{\mathrm{i}} \\
\end{array}$ & $\stackrel{8}{-}$ & $\stackrel{8}{-}$ \\
\hline$x_{l} p_{S}$ & $\stackrel{8}{8}$ & : & : & !n & : & in & 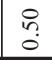 & in & $\stackrel{8}{\square}$ & $\begin{array}{l}0 \\
n \\
0\end{array}$ & $\begin{array}{l}0 \\
n \\
0\end{array}$ & : & $\begin{array}{l}0 \\
n \\
0\end{array}$ & $\stackrel{8}{-}$ & $\stackrel{8}{-}$ & $\stackrel{8}{-}$ & $\underset{i}{\stackrel{一}{i}}$ & $\underset{\mathrm{i}}{\stackrel{\mathrm{i}}{ }}$ \\
\hline $\operatorname{snl} 6100$ & 8 & 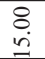 & $\begin{array}{l}8 \\
\dot{8} \\
12\end{array}$ & 8 & 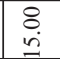 & $\begin{array}{l}8 \\
\\
1 \\
1\end{array}$ & $\begin{array}{l}8 \\
\\
1 \\
1\end{array}$ & $\begin{array}{l}8 \\
8 \\
1 \\
1\end{array}$ & $\underset{8}{8}$ & $\begin{array}{l} \\
\\
0\end{array}$ & $\underset{8}{8}$ & $\begin{array}{l}8 \\
\\
i\end{array}$ & \begin{tabular}{|l|}
8 \\
\\
\end{tabular} & $\begin{array}{l}8 \\
\\
i\end{array}$ & $\begin{array}{l}8 \\
i \\
1\end{array}$ & $\begin{array}{l}8 \\
i \\
i\end{array}$ & 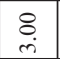 & $\underset{8}{8}$ \\
\hline snu!xp., & $\stackrel{8}{8}$ & $\stackrel{8}{i}$ & $\underset{i}{\stackrel{i}{i}}$ & $\stackrel{8}{8}$ & \begin{tabular}{l}
\multirow{2}{*}{} \\
\end{tabular} & \begin{tabular}{l}
\multirow{2}{*}{} \\
\end{tabular} & \begin{tabular}{l}
\multirow{8}{*}{} \\
in
\end{tabular} & $\begin{array}{l}8 \\
\\
\end{array}$ & 过 & \begin{tabular}{|l|}
\multirow{i}{*}{} \\
\end{tabular} & \begin{tabular}{l}
\multirow{i}{*}{} \\
\end{tabular} & 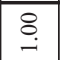 & $\stackrel{8}{8}$ & $\begin{array}{l} \\
\vdots \\
0\end{array}$ & $\begin{array}{l}0 \\
0 \\
0\end{array}$ & \begin{tabular}{ll}
\multirow{2}{*}{} \\
0 \\
0
\end{tabular} & $\begin{array}{ll} \\
\vdots \\
0\end{array}$ & $\begin{array}{l}0 \\
\vdots \\
0\end{array}$ \\
\hline рипәд & 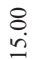 & 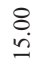 & $\begin{array}{l}8 \\
\qquad \\
1\end{array}$ & $\begin{array}{l}\stackrel{8}{\circ} \\
\stackrel{0}{1}\end{array}$ & $\begin{array}{l}8 \\
\dot{0} \\
\end{array}$ & $\begin{array}{l}8 \\
\dot{0}\end{array}$ & $\begin{array}{l}8 \\
0 \\
0\end{array}$ & $\stackrel{8}{\stackrel{8}{2}}$ & $\begin{array}{l}8 \\
\dot{1}\end{array}$ & \begin{tabular}{l}
$\stackrel{8}{0}$ \\
\hdashline
\end{tabular} & $\stackrel{8}{\stackrel{8}{r}}$ & $\begin{array}{c}8 \\
\underset{1}{2}\end{array}$ & $\begin{array}{l}8 \\
\stackrel{0}{\oplus}\end{array}$ & 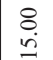 & 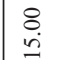 & $\begin{array}{l}8 \\
i \\
-1\end{array}$ & $\begin{array}{l}\underset{8}{8} \\
i \\
-\end{array}$ & $\begin{array}{l}8 \\
\stackrel{0}{9}\end{array}$ \\
\hline $\operatorname{snu}_{I}$ & 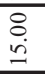 & $\begin{array}{l}8 \\
\dot{i}\end{array}$ & $\begin{array}{l}8 \\
\\
2 \\
2\end{array}$ & $\begin{array}{l}8 \\
\text { in }\end{array}$ & \begin{tabular}{|l|}
8 \\
$\dot{1}$
\end{tabular} & $\begin{array}{l}8 \\
8 \\
1\end{array}$ & \begin{tabular}{|l|}
8 \\
iे
\end{tabular} & $\begin{array}{c}8 \\
1 \\
1\end{array}$ & $\begin{array}{c}8 \\
i \\
i\end{array}$ & \begin{tabular}{|c}
8 \\
$\dot{1}$ \\
\end{tabular} & $\begin{array}{c}8 \\
6 \\
1 \\
-1\end{array}$ & $\begin{array}{c}8 \\
1 \\
1 \\
\end{array}$ & $\begin{array}{c}8 \\
1 \\
1 \\
\end{array}$ & \begin{tabular}{|l|}
8 \\
$\stackrel{1}{1}$
\end{tabular} & $\begin{array}{l}8 \\
\stackrel{4}{1}\end{array}$ & $\begin{array}{l} \\
\\
\dot{1}\end{array}$ & $\begin{array}{l} \\
6 \\
1 \\
2\end{array}$ & $\begin{array}{l} \\
0 \\
0\end{array}$ \\
\hline $\operatorname{solqh}$ & $\stackrel{\circ}{\circ}$ & $\stackrel{\circ}{\circ}$ & : & $\stackrel{8}{:}$ & $\underset{-1}{8}$ & : & $\underset{8}{8}$ & $\underset{1}{8}$ & \begin{tabular}{|l|} 
\\
$i$
\end{tabular} & \begin{tabular}{|l|} 
\\
\end{tabular} & \begin{tabular}{|c|} 
\\
$i$ \\
$i$
\end{tabular} & 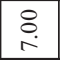 & \begin{tabular}{|l|} 
\\
\\
\end{tabular} & \begin{tabular}{|c|c}
8 \\
1 \\
1
\end{tabular} & $\begin{array}{l}8 \\
i \\
1\end{array}$ & $\begin{array}{l}8 \\
\dot{8} \\
i\end{array}$ & $\underset{j}{\stackrel{8}{n}}$ & $\begin{array}{l}8 \\
i n\end{array}$ \\
\hline $.120 \mathrm{~V}$ & $\begin{array}{l}0 \\
\vdots \\
0 \\
0\end{array}$ & $\begin{array}{ll}0 \\
\vdots \\
0\end{array}$ & $\begin{array}{ll}0 \\
\vdots \\
0\end{array}$ & $\begin{array}{ll}0 \\
\vdots \\
0\end{array}$ & \begin{tabular}{|l|l}
\multirow{2}{*}{} \\
0
\end{tabular} & \begin{tabular}{|l} 
\\
0 \\
0
\end{tabular} & \begin{tabular}{|l|l} 
\\
$\vdots$ \\
0
\end{tabular} & 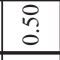 & \begin{tabular}{|l|l} 
\\
$\vdots$ \\
0
\end{tabular} & \begin{tabular}{|l|} 
\\
$\vdots$ \\
0 \\
\end{tabular} & \begin{tabular}{|l|} 
\\
$\vdots$ \\
0 \\
\end{tabular} & \begin{tabular}{|l|} 
\\
0 \\
0
\end{tabular} & \begin{tabular}{|l|}
0 \\
0 \\
\end{tabular} & \begin{tabular}{|l|} 
\\
\\
\end{tabular} & $\begin{array}{l}\circ \\
\\
\end{array}$ & $\frac{9}{\circ}$ & \begin{tabular}{|l|l}
0 \\
$\vdots$ \\
0
\end{tabular} & $\stackrel{\circ}{\circ}$ \\
\hline $\operatorname{sns}_{0} v_{H}$ & in & : & : & $\stackrel{8}{-}$ & $\begin{array}{l}8 \\
i \\
i\end{array}$ & $\underset{-}{\stackrel{一}{*}}$ & 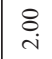 & $\begin{array}{l}8 \\
\text { in }\end{array}$ & $\underset{j}{\stackrel{r}{r}}$ & $\begin{array}{c}8 \\
i \\
i\end{array}$ & 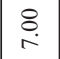 & $\begin{array}{c}8 \\
i \\
i\end{array}$ & \begin{tabular}{|l}
8 \\
8 \\
1
\end{tabular} & 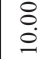 & $\stackrel{8}{\circ}$ & 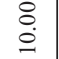 & $\begin{array}{l}\stackrel{8}{0} \\
\stackrel{0}{\circ}\end{array}$ & i \\
\hline snpndo $_{d}$ & $\overline{0}$ & $\overrightarrow{0}$ & $\overline{0}$ & $\overline{0}$ & $\overrightarrow{0}$ & $\overrightarrow{0}$ & $\overline{0}$ & $n$ & $\overrightarrow{0}$ & $\because$ & $\overrightarrow{0}$ & $n$ & $\overrightarrow{0}$ & ? & 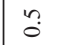 & $n$ & $\overrightarrow{0}$ & $n$ \\
\hline$[x] \Gamma_{L}$ & 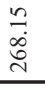 & $\begin{array}{l}n \\
0 \\
\stackrel{0}{0} \\
\end{array}$ & 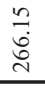 & 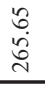 & 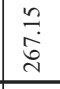 & \begin{tabular}{|l}
$n$ \\
0 \\
0 \\
1 \\
\end{tabular} & \begin{tabular}{|l|}
$n$ \\
0 \\
0 \\
\\
\end{tabular} & \begin{tabular}{|l|l} 
& 0 \\
0 \\
0 \\
1 \\
\end{tabular} & \begin{tabular}{|l}
0 \\
0 \\
6 \\
$i$ \\
\end{tabular} & \begin{tabular}{|l|l} 
\\
0 \\
0 \\
$ن$ \\
\end{tabular} & \begin{tabular}{|l|} 
\\
0 \\
8 \\
$i$ \\
\end{tabular} & \begin{tabular}{|l|}
$n$ \\
0 \\
8 \\
$i$ \\
\end{tabular} & \begin{tabular}{|l|l} 
\\
0 \\
0 \\
0 \\
1 \\
\end{tabular} & 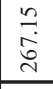 & 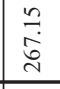 & 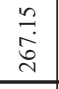 & $\begin{array}{l}n \\
0 \\
0 \\
0 \\
1\end{array}$ & $\begin{array}{l}\frac{n}{0} \\
\stackrel{0}{0} \\
\end{array}$ \\
\hline $\operatorname{sWV} \mathrm{dq} \supset_{t \downarrow 1}$ & ָָ & \&े & $\begin{array}{l}\text { तิ } \\
\text { }\end{array}$ & $\begin{array}{l}\tilde{\sigma} \\
\tilde{\sigma}\end{array}$ & $\begin{array}{l}\stackrel{\ominus}{7} \\
\end{array}$ & \begin{tabular}{|l|}
\multicolumn{1}{c}{} \\
f \\
\end{tabular} & 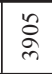 & $\begin{array}{l}a \\
a \\
m\end{array}$ & 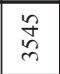 & \begin{tabular}{|l|}
$n$ \\
$\hat{n}$ \\
$\tilde{n}$
\end{tabular} & 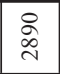 & \begin{tabular}{l} 
\\
\multirow{d}{*}{} \\
\end{tabular} & $\begin{array}{l} \\
\frac{\pi}{N}\end{array}$ & 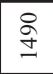 & 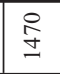 & 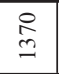 & 吕 & - \\
\hline
\end{tabular}


Results of Principal Components Analysis (PCA) for skulls and mandibles of Bechstein's bat.

\begin{tabular}{|c|c|c|c|c|c|}
\hline \multicolumn{3}{|c|}{ Skulls N=190 } & \multicolumn{3}{|c|}{ Mandibles $\mathrm{N}=212$} \\
\hline $\mathrm{PC}$ & Eigenvalue & $\%$ variance & $\mathrm{PC}$ & Eigenvalue & $\%$ variance \\
\hline 1 & 0.500675 & 49.384 & 1 & 0.00114 & 39.061 \\
\hline 2 & 0.160075 & 15.789 & 2 & 0.000977 & 33.503 \\
\hline 3 & 0.074495 & 7.3477 & 3 & 0.000314 & 10.756 \\
\hline 4 & 0.048561 & 4.7897 & 4 & 0.000297 & 10.183 \\
\hline 5 & 0.039849 & 3.9305 & 5 & 0.000137 & 4.7084 \\
\hline 6 & 0.029704 & 2.9298 & 6 & $5.22 \mathrm{E}-05$ & 1.7883 \\
\hline 7 & 0.029098 & 2.87 & & & \\
\hline 8 & 0.024197 & 2.3866 & & & \\
\hline 9 & 0.023374 & 2.3054 & & & \\
\hline 10 & 0.021653 & 2.1357 & & & \\
\hline 11 & 0.016346 & 1.6123 & & & \\
\hline 12 & 0.015628 & 1.5415 & & & \\
\hline 13 & 0.012366 & 1.2197 & & & \\
\hline 14 & 0.010117 & 0.99784 & & & \\
\hline 15 & 0.007712 & 0.76068 & & & \\
\hline
\end{tabular}

Results of Discriminant Analysis (DA) for skulls and mandibles of Bechstein's bat.

\begin{tabular}{|c|c|c|c|c|c|}
\hline \multicolumn{3}{|c|}{ Skulls N=179 } & \multicolumn{3}{|c|}{ Mandibles N=198 } \\
\hline Parameter & $\mathrm{F}$ & $\mathrm{p}$ & Parameter & $\mathrm{F}$ & $\mathrm{p}$ \\
\hline GLS & 6.2874 & 0.0000 & MDL & 5.45687 & 0.00000 \\
\hline CLS & 4.6849 & 0.0000 & MBTR & 2.88447 & 0.00229 \\
\hline $\mathrm{PM}_{1}$ & 1.4129 & 0.1793 & LPA & 5.67441 & 0.00000 \\
\hline MSW & 3.4676 & 0.0004 & $\mathrm{HCP}$ & 8.72381 & 0.00000 \\
\hline MZB & 7.9880 & 0.0000 & $\mathrm{HP}_{4}$ & 6.47495 & 0.00000 \\
\hline PSW & 6.9799 & 0.0000 & $\mathrm{HM}_{3}$ & 6.84590 & 0.00000 \\
\hline $\mathrm{COW}$ & 7.2200 & 0.0000 & & & \\
\hline TPL & 7.8801 & 0.0000 & & & \\
\hline $\mathrm{L}\left(\mathrm{C}-\mathrm{P}_{3}\right)$ & 5.0741 & 0.0000 & & & \\
\hline LTR & 6.1599 & 0.0000 & & & \\
\hline $\mathrm{L}\left(\mathrm{P}_{4}-\mathrm{M}_{3}\right)$ & 2.3565 & 0.0128 & & & \\
\hline$L\left(M_{1}-M_{3}\right)$ & 9.6325 & 0.0000 & & & \\
\hline $\mathrm{PC}$ & 5.2394 & 0.0000 & & & \\
\hline FCW & 2.3864 & 0.0117 & & & \\
\hline $\mathrm{L}\left(\mathrm{P}_{1}-\mathrm{P}_{3}\right)$ & 13.6642 & 0.0000 & & & \\
\hline
\end{tabular}


Environmental factors correlated with Bechstein's bat morphology.

\begin{tabular}{|c|l|}
\hline Nr. & Parameter \\
\hline 1 & Age of sample $\left({ }^{14}\right.$ C bp) \\
\hline 2 & Climate \\
\hline 3 & Mean temperature of January (T_j) \\
\hline 4 & Mean annual temperatute (T_y) \\
\hline 5 & High above sea level (H_a.s.1.) \\
\hline 6 & Floods \\
\hline 7 & Frequency of floods (Fr_floods) \\
\hline 8 & Frequency of the species \\
\hline 9 & Fir Abies alba \\
\hline 10 & Maple Acer \\
\hline 11 & Alder Alnus \\
\hline 12 & Birch Betula \\
\hline 13 & Hornbeam Carpinus betulus \\
\hline 14 & Hazel Corylus avellana \\
\hline 15 & Beech Fagus silvatica \\
\hline 16 & Ash Fraxinus excelsior \\
\hline 17 & Spruce Picea abies \\
\hline 18 & Pine Pinus \\
\hline 19 & Oak Quercus \\
\hline 20 & Willow Salix \\
\hline 21 & Lime Tilia \\
\hline 22 & Elm Ulmus \\
\hline 23 & Poplar and aspen Populus \\
\hline 24 & Artemisia \\
\hline 25 & Sedge family Cyperaceae \\
\hline 26 & Grass family Poaceae (Gramineae) \\
\hline 27 & Bulrush (cat-tail) Typha latifolia \\
\hline 28 & Herbs \\
\hline & \\
\hline
\end{tabular}

Figures 3-12 presents microevolution trends of cranial dimensions, with the strongest discrimination to the groups. Despite the fact that differences between groups are mostly not statistically significant, microevolutionary trends in morphology are visible. Changes of each parameter has individual course, but trends are mostly similar. GLS (Fig. 3), COW (Fig. 6), LTR (Fig. 8), and HCP (Fig. 10) increase espacially during Subatlantic period (from $2800{ }^{14} \mathrm{C}$ BP till recent time). At the same time MZB (Fig. 4), PSW (Fig. 5), and $\mathrm{HM}_{3}$ (Fig. 12) decrease. Some of cranial parameters: TPL, $\mathrm{L}\left(\mathrm{M}_{1}-\mathrm{M}_{3}\right)$, and $\mathrm{HP}_{4}$ seem to be rather stable (Figs $7,9,11$ ).

During the Subboreal period $\left(5115-2800{ }^{14} \mathrm{C}\right.$ BP) four environmental variables have the strongest influence on skull morphology of investigated species. Those are: age of sample, mean temperature of January, and percentage of Populus and Fagus silvatica in vegetation (Fig. 13, tab. 8). In the same time range another four variables correlates significantly with mandible morphology: presence of floods, percentage of Tilia in vegetation, and frequency of $M$. bechsteinii and ecologically close species $-M$. nattereri (Fig. 15, tab. 9). 


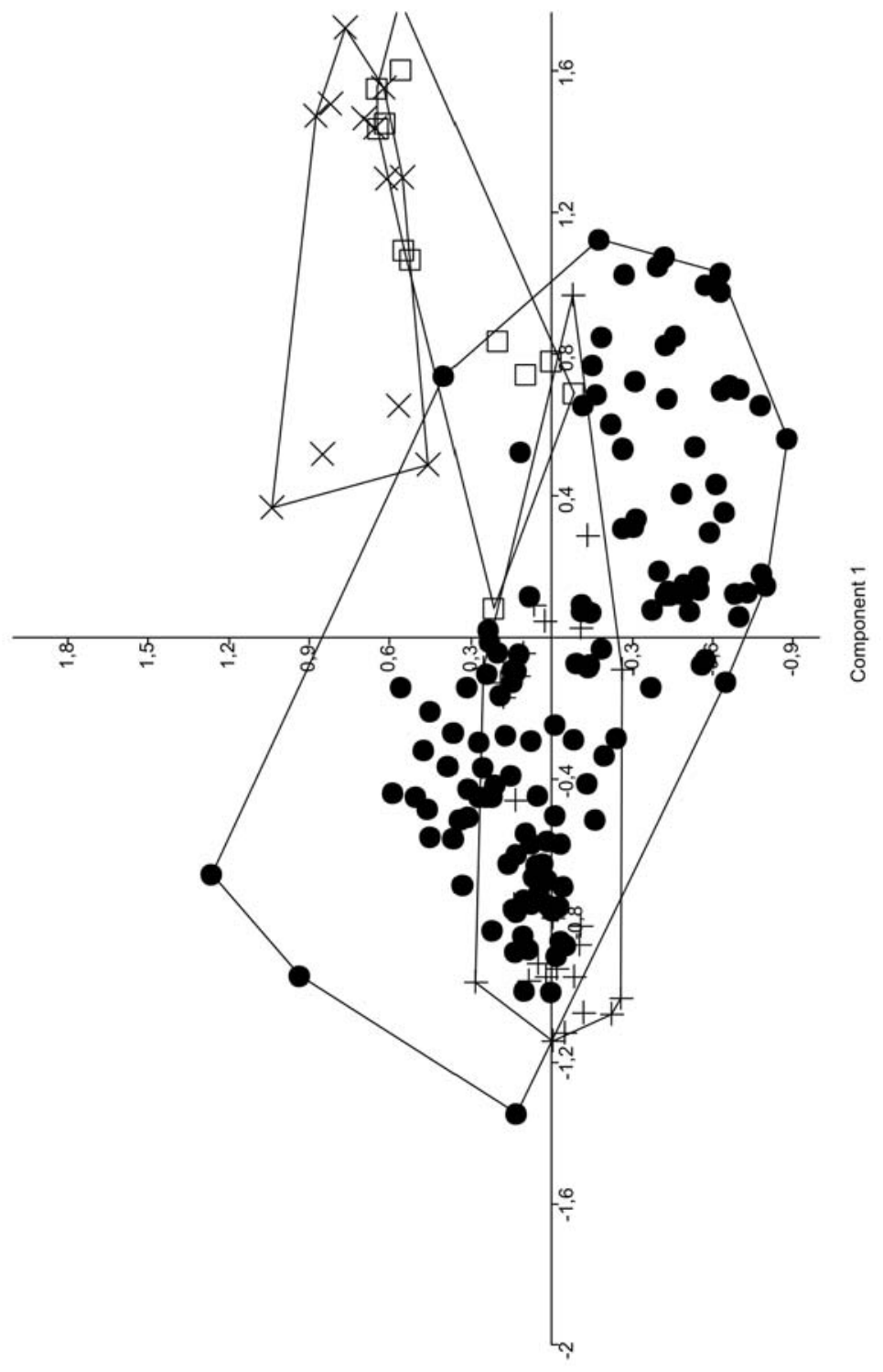

Z juəuodwoว
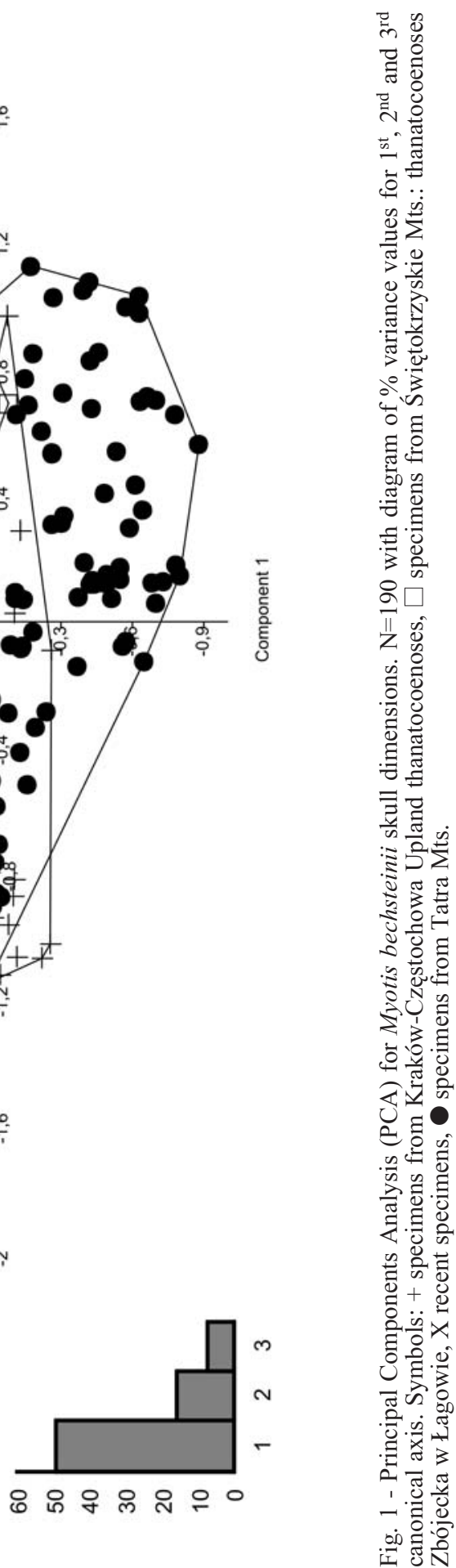


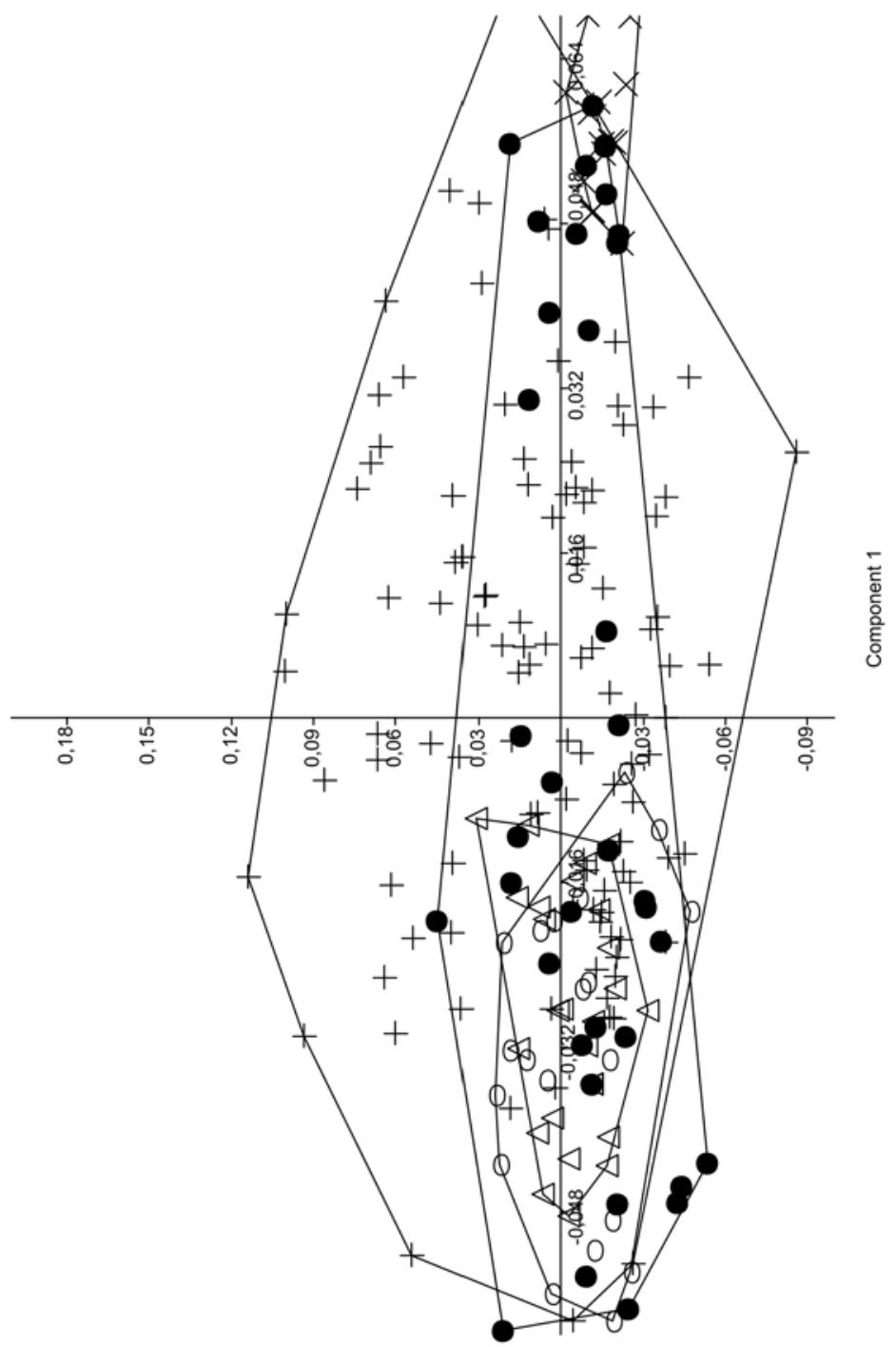

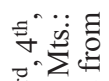

m.

를

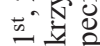

t.

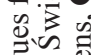

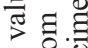

80

. 들 के

సँ. छ

苍 क्षे

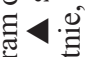

की

동

$38 . \overline{0}$

글

乙 元密

氜它

늘

寻

흥 능

콩

:초일

氙

$\approx$ क

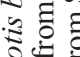

I.

훙.

\&.

要

a

2 \}uәиodmoว

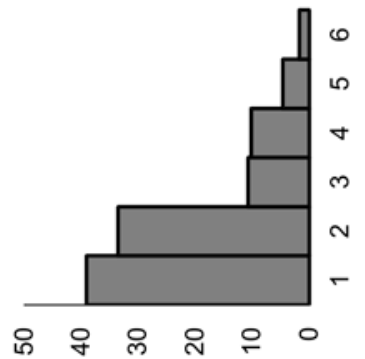

플

考

的

可.

을

듀유요

등 ำ

.

눙요

글

旺品艺 
Table 8

Results of gradient selection in Redundancy Analysis (RDA) for cranial skeleton of Bechstein's bat.

\begin{tabular}{|c|c|c|c|c|c|c|c|}
\hline & & Factor & Lambda & $\mathrm{r}$ & $\mathrm{p}$ & $\mathrm{F}$ & $\begin{array}{c}\mathrm{Nr} \text { of } \\
\text { permutations }\end{array}$ \\
\hline Skulls & Subboreal & Age of sample & 0.03 & -0.7536 & 0.004 & 3.88 & 499 \\
\hline & & $\mathrm{T}$ january & 0.03 & 0.7175 & 0.004 & 3.88 & 499 \\
\hline & & Populus & 0.03 & 0.1735 & 0.004 & 3.88 & 499 \\
\hline & & Fagus silvatica & 0.03 & 0.6147 & 0.004 & 3.88 & 499 \\
\hline & Subatlantic & Age of sample & 0.01 & -0.3976 & 0.002 & 2.29 & 499 \\
\hline & & $\mathrm{T}$ january & 0.01 & 0.8194 & 0.002 & 2.29 & 499 \\
\hline & & Populus & 0.01 & -0.0532 & 0.002 & 2.29 & 499 \\
\hline & & Fagus silvatica & 0.01 & -0.2918 & 0.002 & 2.29 & 499 \\
\hline & & Frequency_MBE & 0.01 & -0.4454 & 0.002 & 2.29 & 499 \\
\hline Mandibles & Subboreal & Floods & 0.12 & 0.5296 & 0.002 & 14.4 & 499 \\
\hline & & Frequency_MBE & 0.1 & -0.3459 & 0.002 & 13.1 & 499 \\
\hline & & Tilia & 0.08 & 0.2142 & 0.002 & 11.3 & 499 \\
\hline & & Frequency_MYN & 0.02 & 0.2700 & 0.002 & 3.72 & 499 \\
\hline & Subatlantic & Fraxinus excelsior & 0.62 & 0.9443 & 0.002 & 84.91 & 499 \\
\hline & & H_a.s.l. & 0.03 & 0.1872 & 0.004 & 4.13 & 499 \\
\hline
\end{tabular}

In Subatlantic period $\left(2800{ }^{14} \mathrm{C}\right.$ BP till now $)$ five factors influence on skull morphology: age of sample, mean temperature of January, percentage of Populus and Fagus silvatica in vegetation, and frequency of M. bechsteinii (Fig. 14, tab. 8). The same time range characterizes correlation of hight a.s.l. and percentage of Fraxinus excelsior in vegetation with mandible morphology of Bechstein's bat (Fig. 16, tab. 8).

Mean temperature of January, percentage of $F$. excelsior, frequency of Bechstein's bat and occurence of floods determine morphology changes the most (Figs 13-16).

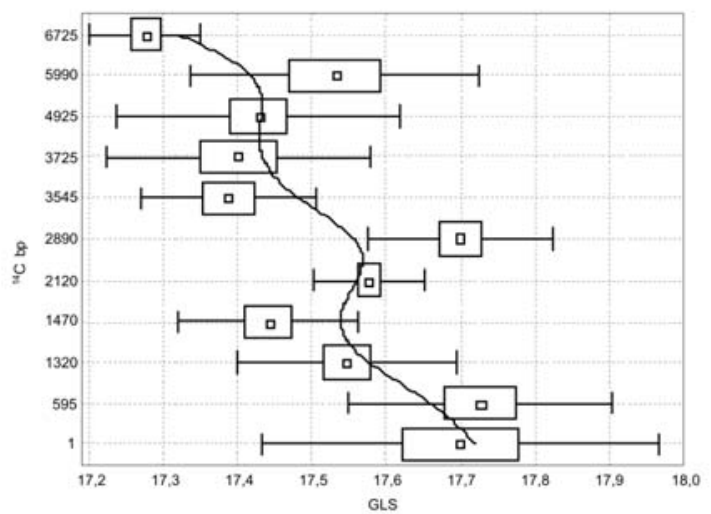

Fig. 3 - Changes in greatest lenght of skull (GLS) (in mm) of Myotis bechsteinii in the Middle and Late Holocene. (中-Mean; $\square$ - Mean \pm Standard error; $\longmapsto$ - Mean \pm Standard deviation) 


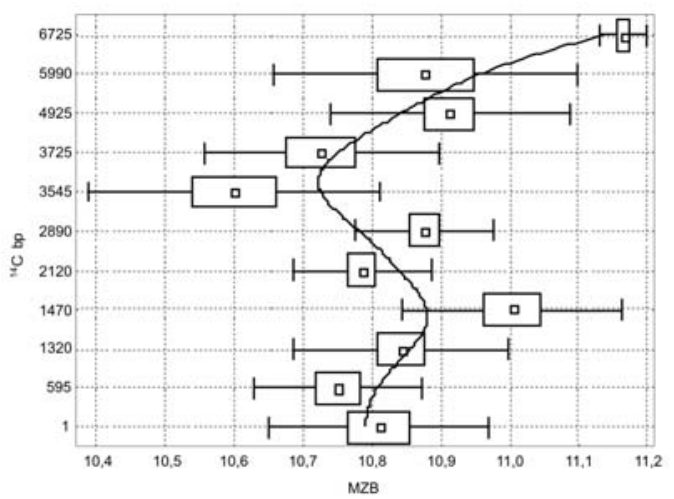

Fig. 4 - Changes in maximum zygomatic breadth (MZB) (in mm) of Myotis bechsteinii in the Middle and Late Holocene. ( $\$$ - Mean; $\square$ - Mean \pm Standard error; $\longmapsto$ - Mean \pm Standard deviation)

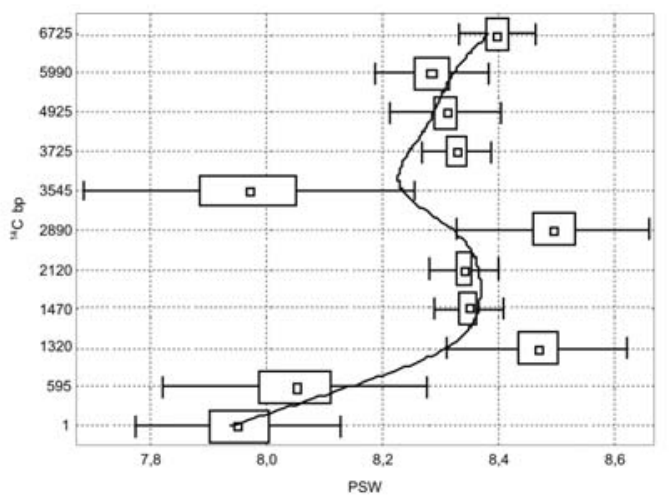

Fig. 5 - Changes in posterior skull width (PSW) (in mm) of Myotis bechsteinii in the Middle and Late Holocene. (中-Mean; $\square$ - Mean \pm Standard error; $\longmapsto$ - Mean \pm Standard deviation)

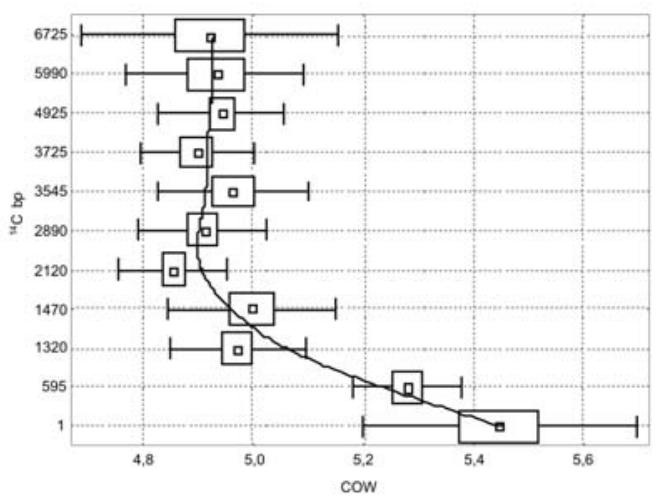

Fig. 6 - Changes in condylus occipitalis width (COW) (in mm) of Myotis bechsteinii in the Middle and Late Holocene. (ф- Mean; $\square$ - Mean \pm Standard error; $\mapsto$ - Mean \pm Standard deviation) 


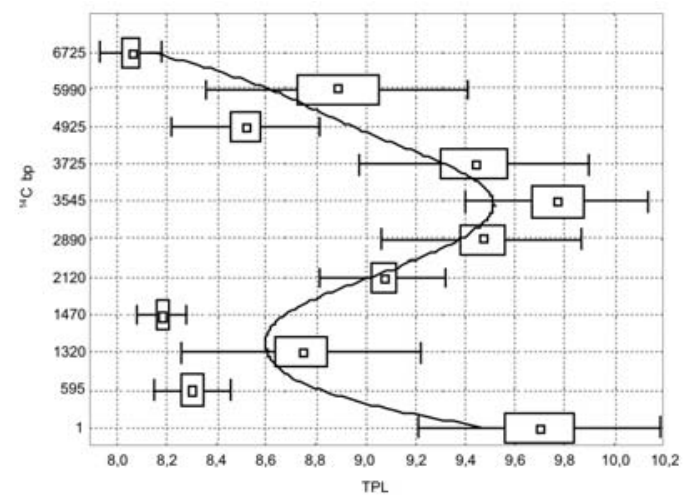

Fig. 7 - Changes in total palate lenght (TPL) (in mm) of Myotis bechsteinii in the Middle and Late Holocene. (中-Mean; $\square$ - Mean \pm Standard error; $\longmapsto$ - Mean \pm Standard deviation)

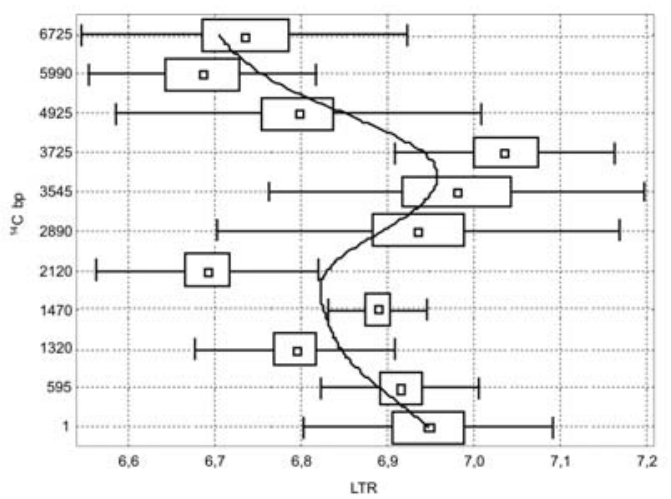

Fig. 8 - Changes in toothrow lenght (LTR) (in mm) of Myotis bechsteinii in the Middle and Late Holocene. (中- Mean; $\square$ - Mean \pm Standard error; $\mapsto$ - Mean \pm Standard deviation)

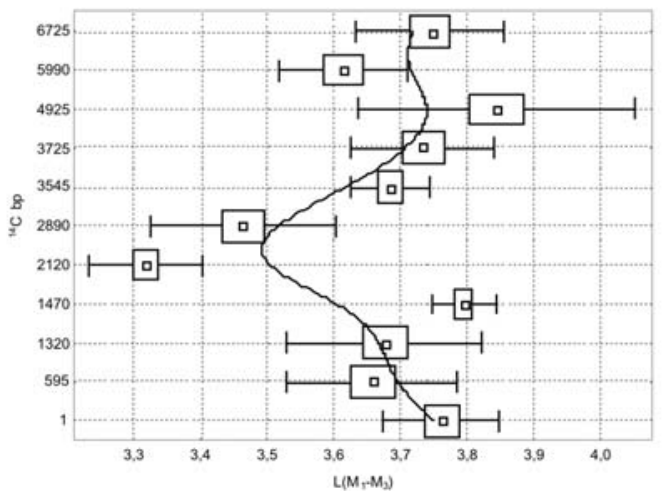

Fig. 9 - Changes in $\mathrm{M}_{1}$ to $\mathrm{M}_{3}$ lenght $\left(\mathrm{L}\left(\mathrm{M}_{1}-\mathrm{M}_{3}\right)\right.$ ) (in mm) of Myotis bechsteinii in the Middle and Late Holocene. (ф- Mean; $\square$ - Mean \pm Standard error; $\mapsto$ - Mean \pm Standard deviation) 


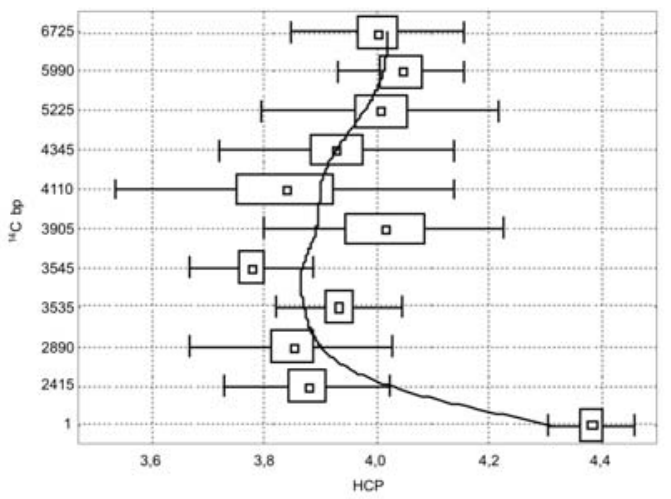

Fig. 10 - Changes in height of coronoid process (HCP) (in mm) of Myotis bechsteinii in the Middle and Late Holocene. (ф- Mean; $\square$ - Mean \pm Standard error; $\mapsto$-Mean \pm Standard deviation)

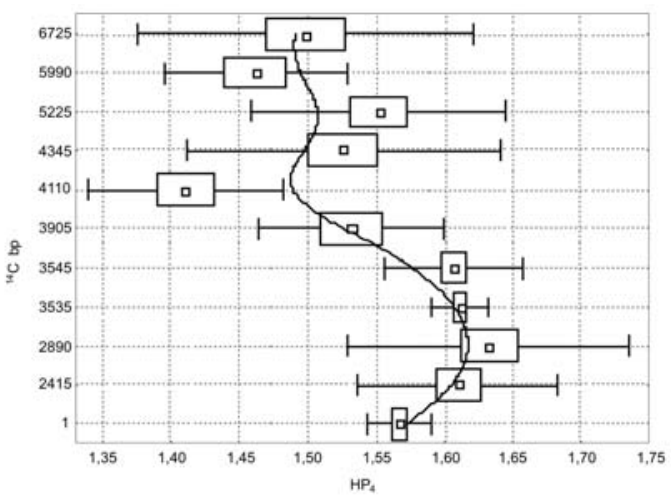

Fig. 11 - Changes in height of mandible at $\mathrm{P}_{4}\left(\mathrm{HP}_{4}\right)$ (in $\mathrm{mm}$ ) of Myotis bechsteinii in the Middle and Late Holocene. (申- Mean; $\square$ - Mean \pm Standard error; - - Mean \pm Standard deviation)

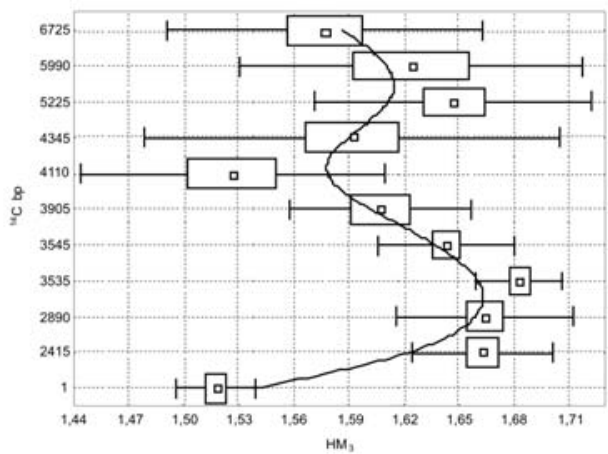

Fig. 12 - Changes in height of mandible at $\mathrm{M}_{3}\left(\mathrm{HM}_{3}\right)$ (in $\mathrm{mm}$ ) of Myotis bechsteinii in the Middle and Late Holocene. (ф- Mean; $\square$ - Mean \pm Standard error; -1 - Mean \pm Standard deviation) 


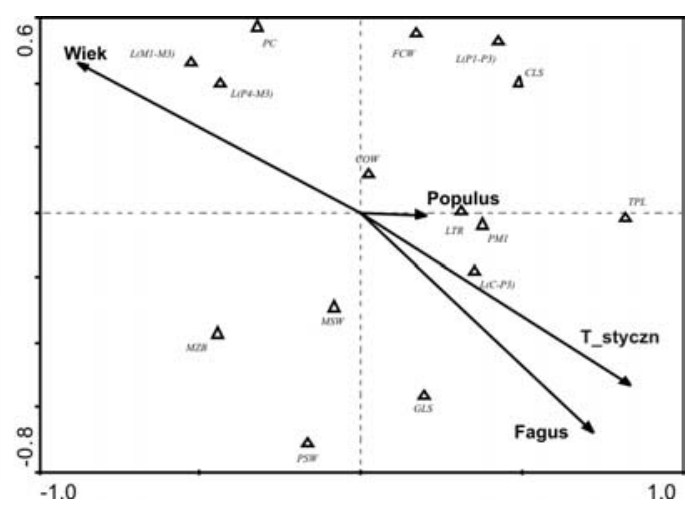

Fig. 13 - Diagram of Bechstein's bat skull dimensions in gradient of environmental factors having signifiant influence into morphology during the Subboreal period $\left(5115-2800{ }^{14} \mathrm{C} \mathrm{BP}\right)$ based on gradient selection redundancy analysis (RDA).

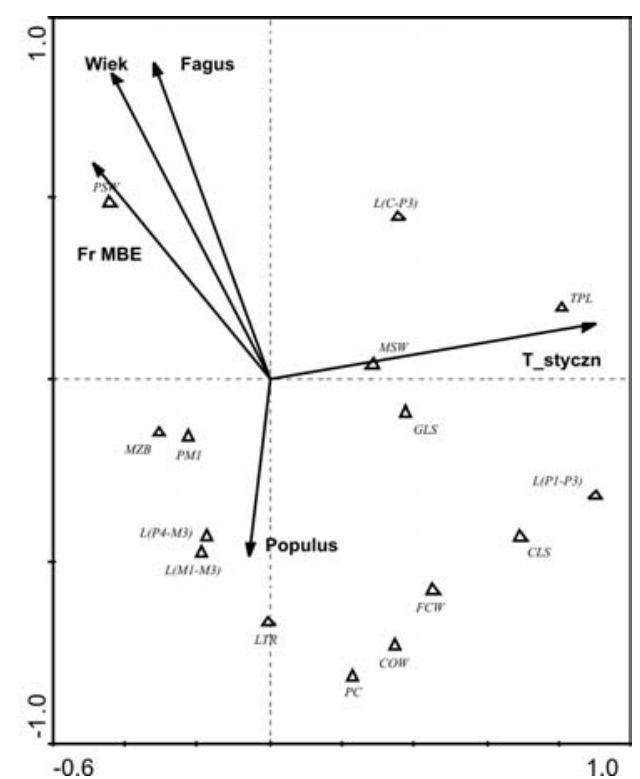

Fig. 14 - Diagram of Bechstein's bat skull dimensions in gradient of environmental factors having signifiant influence into morphology during the Subatlantic period $\left(2800{ }^{14} \mathrm{C}\right.$ BP till now $)$ based on gradient selection redundancy analysis (RDA).

\section{DISCUSSIONS}

Bats are the only mammals able to active flight (Sachanowicz \& Ciechanowski, 2005). This ability determining cranial morphology of bats as well as changes in climate, vegetation feedback and all modifications in ecological niche of the species (Horáček, 1976; Kordos, 1982; Obuch, 1995; Zahn, 1999).

During the Middle and Late Holocene cranial morphology of Bechstein's bat shows the following trends: greatest lenght of skull, condylus occipitalis width, tooth row length, and hight of coronoid process increase. At the same time maximum 


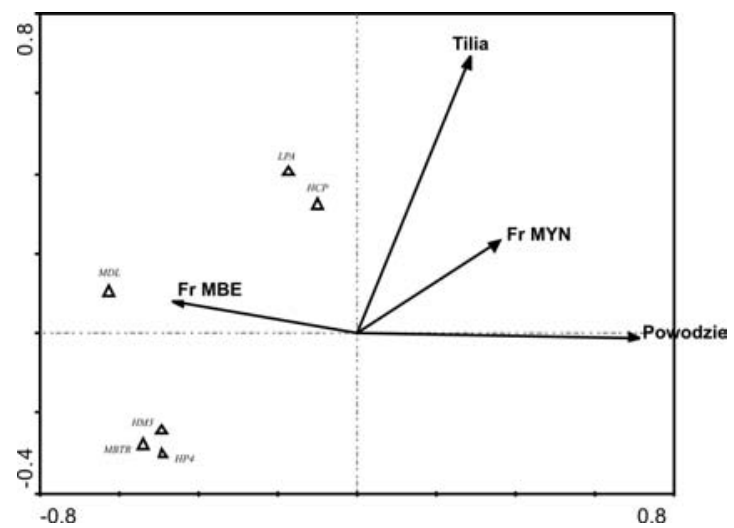

Fig. 15 - Diagram of Bechstein's bat mandible dimensions in gradient of environmental factors having signifiant influence into morphology during the Subboreal period $\left(5115-2800{ }^{14} \mathrm{C} \mathrm{BP}\right)$ based on gradient selection redundancy analysis (RDA).

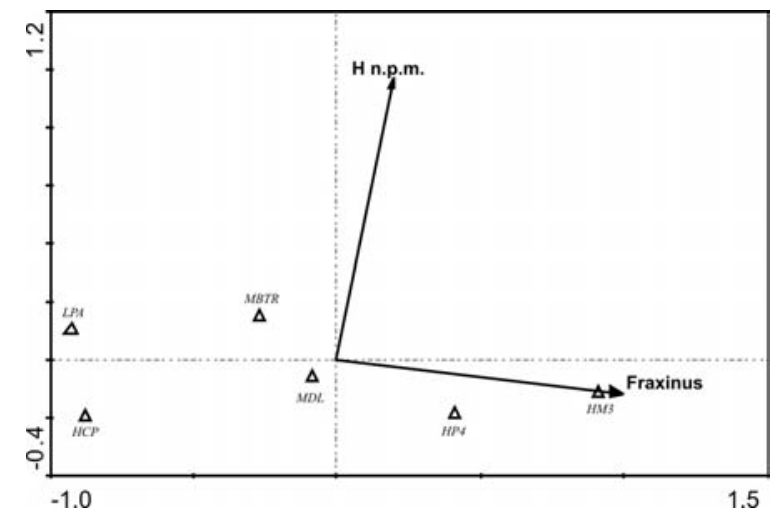

Fig. 16 - Diagram of Bechstein's bat mandible dimensions in gradient of environmental factors having signifiant influence into morphology during the Subatlantic period $\left(2800{ }^{14} \mathrm{C}\right.$ BP till now $)$ based on gradient selection redundancy analysis (RDA).

zygomatic breadth, posterior skull width and hight of mandible ramus under third molar decrease, while total palate length, lenght from the first to the third molar, and hight of mandible ramus under fourth premolar seem to be rather stable.

Those are trends in making cranial skeleton more slender, skull seem to became narrower and longer. Place of muscle attachment seem to became wider. Molar row lenght rather does not change, but skull and mandible became longer, being possible the effect of extending premolar row length. Surely, all these trends are the effect of optimazing the skeleton proportions to the muscle mass, which are saved, when there is assured high effectiveness of flight and biting of food, and low body mass at the same time (Czarnecki \& Kallen, 1980; Smith \& Savage, 1956). Presented microevolutionary trends in cranial skeleton of this species can possibly be part of long-term modifications, or reversable changes as morphological answer to the climate and ecological niche fluctuations (Futuyma, 2008).

Influence of sexual dimorphism to differences between populations of $M$. bechsteinii from radiocarbon dated thanatocoenoses are excluded by researching 
recent population of the species. Differences between them can be rather a result coming from varied geographical regions (mountains, or uplands of southern Poland), where climatic changes and events have individual course.

Ten of 28 investigated environmental factors have signifficant influence on cranial morphology of Bechstein's bat. Influence of the age of sample on skull parameters can mean, that morphological changes are part of long-term directioned modifications (Smith \& Savage, 1956).

Temperature of January is strongly correlated with mean temperature of the year and it's obvious factor, determining presence of the species in region (Krebs, 2001). For bats, which are warm-likely animals, living in colder region can mean worse condition of population. Individuals can be weaker than those from warmer regions (Wimsatt, 1970).

Frequency of investigated bat species, as well as frequency of Natterer's bat, can also be information about condition of population. Probably the higher frequency of Bechstein's bat, the better condition of population. Opposite direction can be with higher frequency of $M$. nattereri, as possible competitor for the same niche.

Percentage of pollen of beech, lime, ash, poplar and aspen gives information about participation of theese species in forests. They correlates with morphology of $M$. bechsteinii possibly as good habitat. Especially beech is known as species important for Bechstein's bat. Old, hollowed trees are inhabitated by this species very often during the summer (Sachanowicz \& Ciechanowski, 2005).

Hight above sea level says a lot about differences between populations from uplands and mountains. It has to be factor determining not only frequency, but also morphology of bats. During the Atlantic and Subboreal period frequency of Bechstein's bat varied from $2-65 \%$ and was higher on uplands. Nowadays this species is almost completely eliminated from Polish mountain fauna (Postawa, 2004; Piksa \& Wołoszyn, 2001).

Occurence of floods is connected with cooler climate of Subboreal period (Starkel, 1999). It is also a resistant factor to the occurence and morphology of $M$. bechsteinii. Neighbourhood of water reservoirs is an ecosystem typical for other bat species (for example Daubenton's bat Myotis daubentonii), not Bechstein's bat (Sachanowicz \& Ciechanowski, 2005). It must be the reason why floods are correlating with morphology of $M$. bechsteinii.

\section{ACKNOWLEDGEMENTS}

Researches conducted thanks to Polish Ministry of Science and Higher Education foundation, project nr. N N303 324836 from $27^{\text {th }}$ of March 2009.

Special thanks to Dr Tomasz Skalski from the Institute of Zoology, Jagiellonian University in Kraków, for consultations and help in statistical analysis.

\section{MICROEVOLUȚIA LILIACULUI LUI BECHSTEIN MYOTIS BECHSTEINII (KUHL, 1817) (MAMMALIA: CHIROPTERA), ÎN HOLOCENUL POLONIEI DE SUD}

\section{REZUMAT}

S-au studiat tendințele microevoluționare ale craniului liliacului lui Bechstein. În ciuda faptului că diferențele dintre grupuri nu sunt deosebite din punct de vedere statistic, tendințele microevoluţionare morfologice sunt vizibile. Schimbările fiecărui parametru au un curs individual, dar tendințele sunt aproape similare. 
Analiza statistică a facut posibil răspunsul la întrebarea care din factorii de nișe au o influență importantă la morfologia craniului și care este direcția acestei influențe. Temperatura medie a lunii ianuarie, procentul lui Fraxinus excelsior, frecvența liliacului lui Bechstein şi frecvența inundațiilor determină cel mai mult schimbările morfologice.

\section{LITERATURE CITED}

BECK, A., 1995 - Fecal analysis of European bat species. Myotis, 32-33: 109-119.

BOGDANOWICZ, W., 1992 - Sexual dimorphism in size of the skull in European Myotis daubentoni (Mammalia: Chiroptera). Prague Studies in Mammalogy, Praha: 17-25.

CHEDDALI, R., A. BAR-HEN, 2009 - Spatial gradient of temperature and potential vegetation feedback across Europe during the late Quaternary. Climatic Dynamics, 32: 371-379.

CZARNECKI, R. T., F. C. KALLEN, 1980 - Craniofacial, occlusal, and masticatory anatomy in bats. The Anatomical Record, 198: 87-105.

FENTON, M. B., W. BOGDANOWICZ, 2002 - Relationships between external morphology and foraging behaviour: bats in the genus Myotis. Canadian Journal of Zoology, 80: 10041013.

FUTUYMA, D. J. [red. Radwan J.], 2008 - Ewolucja. Wydawnictwo Uniwersytetu Warszawskiego, Warszawa, 606 pp. (in Polish)

HORAČEK, I., 1976 - Přehled kvatérních netopýrů (Chiroptera) Československa. Lynx, 18: 35-58. (in Czech)

KREBS, C. J., 2001 - Ekologia. Eksperymentalna analiza rozmieszczenia i liczebności. Wydawnictwo Naukowe PWN, Warszawa, 735 pp. (in Polish)

KORDOS, L., 1982 - Evolution of the Holocene Vertebrate Fauna in the Carpathian Basin. Zeitschrift fuer Geologische Wissenschaften, 10 (7): 963-970.

LEPŠ, J., P. ŠMILAUER, 1999 - Multivariate Analysis of Ecological Data. Faculty of Biological Sciences, University of South Bohemia České Budějovice, 110 pp.

OBUCH, J., 1995 - Nové poznatky o výskyte nietopierov v jaskinných tanatocenozach. Nietopiere, 1: 29-38. (in Polish)

RAIBle, C. C., C. CASTY, J. LUTERBACHER, A. PAUling, J. ESPER, D. C. FRANK, U. BÜNTGEN, A. C. ROESH, P. TSCHUCK, M. WILD, P. VIDALE, C. SCHÄR, H. WANNER, 2006 - Climate variability - observations, reconstructions, and model simulations for the Atlantic - European and Alpine region from 1500-2100 AD. Climatic Change, 79: 9-29.

RALSKA-JASIEWICZOWA, M. [ed.], M. LATAŁOWA, K. WASYLIKOWA, K. TOBOLSKI, E. MADEYSKA, H. E. WRIGHT Jr., C. TURNER, D. NALEPKA, A. WALANUS, 2004 - Late Glacial and Holocene history of vegetation in Poland based on isopollen maps. W. Szafer Institute of Botany, Polish Academy of Sciences, Kraków: 11-425.

PAWŁOWSKI, J., 1999 - Przemiany fauny od pleniglacjału do czasów współczesnych. Pp: 159-175. In: L. Starkel (ed.), Geografia Polski. Środowisko Przyrodnicze, PWN. Warszawa, 570 pp. (in Polish)

PIKSA, K., B. W. WOŁOSZYN, 2001 - Postglacial bat remains from the Polish Tatra Caves. Lynx, 32: 301-311.

POSTAWA, T., 2004 - Changes in bat fauna during the Middle and Late Holocene as exemplified by thanatocoenoses dated with 14C AMS from Kraków-Częstochowa Upland caves, Poland. Acta Chiropterologica, 6 (2): 269-292.

SACHANOWICZ, K., M. CIECHANOWSKI, 2005 - Nietoperze Polski. Multico Oficyna Wydawnicza. Warszawa: 48-52. (in Polish)

SMITH, J. M., R. J. SAVAGE, 1956 - Some locomotory adaptations in mammals. Zoological Journal of the Linnean Society of London, 42: 603-622.

STARKEL, L. (ed.), 1999 - Geografia Polski, środowisko przyrodnicze. Wydawnictwo Naukowe PWN. Warszawa: 81-569. (in Polish)

TER BRAAK, C. J. F., I. C. PRENTICE, 1988 - A theory of Gradient Analysis. Advances in Ecologycal Research, 18: 271-317. 
WALANUS, A., T. GOSLAR, 2004 - Wyznaczanie wieku metodą ${ }^{14} \mathrm{C}$ dla archeologów, Wydawnictwo Uniwersytetu Rzeszowskiego: 9-114. (in Polish)

WIMSATT, W. A. (ed.), 1970 - Biology of bats. Vol. I. Academic Press Inc., New York. 406 pp.

ZAHN, A., 1999 - Reproductive success, colony size and roost temperature in attic-dwelling bat Myotis myotis. Journal of Zoology (London), 247: 275-280.

Received: January 21, 2010

Accepted: November 30, 2010

\section{Katarzyna Stanik}

International Doctoral Studies in Natural Sciences

at the Polish Academy of Sciences in Kraków.

Institute of Systematics and Evolution of Animals,

Polish Academy of Sciences,

Stawkowska Street 17, 31-016 Kraków, Poland

e-mail: katarzyna.stanik@gmail.com

Bronisław W. Wołoszyn

Chiropterological Information Center, Institute of Systematics and Evolution of Animals, Polish Academy of Sciences,

Stawkowska Street 17, 31-016 Kraków, Poland.

e-mail: woloszbr@isez.pan.krakow.pl 